\title{
Heterotypic seeding of Tau fibrillization by pre-aggregated Abeta provides potent seeds for prion-like seeding and propagation of Tau-pathology in vivo
}

\author{
Bruno Vasconcelos ${ }^{1} \cdot$ Ilie-Cosmin Stancu ${ }^{1} \cdot$ Arjan Buist $^{2} \cdot$ Matthew Bird $^{1} \cdot$ \\ Peng Wang ${ }^{1}$ - Alexandre Vanoosthuyse ${ }^{1} \cdot$ Kristof Van Kolen $^{2} \cdot$ An Verheyen $^{2} \cdot$ \\ Pascal Kienlen-Campard ${ }^{1} \cdot$ Jean$^{-N o e ̈ l ~ O c t a v e ~}{ }^{1} \cdot$ Peter Baatsen $^{3} \cdot$ Diederik Moechars $^{2}$. \\ Ilse Dewachter ${ }^{1}$
}

Received: 19 August 2015 / Revised: 13 December 2015 / Accepted: 13 December 2015 / Published online: 6 January 2016 (c) The Author(s) 2016. This article is published with open access at Springerlink.com

\begin{abstract}
Genetic, clinical, histopathological and biomarker data strongly support Beta-amyloid $(\mathrm{A} \beta)$ induced spreading of Tau-pathology beyond entorhinal cortex (EC), as a crucial process in conversion from preclinical cognitively normal to Alzheimer's Disease (AD), while the underlying mechanism remains unclear. In vivo preclinical models have reproducibly recapitulated $A \beta$-induced Tau-pathology. Tau pathology was thereby also induced by aggregated $A \beta$, in functionally connected brain areas, reminiscent of a prion-like seeding process. In this work we demonstrate, that pre-aggregated $\mathrm{A} \beta$ can directly induce Tau fibrillization by cross-seeding, in a cell-free assay, comparable to that demonstrated before for alpha-synuclein and Tau. We furthermore demonstrate, in a well-characterized cellular Tau-aggregation assay that $\mathrm{A} \beta$-seeds crossseeded Tau-pathology and strongly catalyzed pre-existing Tau-aggregation, reminiscent of the pathogenetic process in AD. Finally, we demonstrate that heterotypic seeded Tau by pre-aggregated $A \beta$ provides efficient seeds for
\end{abstract}

B. Vasconcelos and I.-C. Stancu contributed equally to this work.

Electronic supplementary material The online version of this article (doi:10.1007/s00401-015-1525-x) contains supplementary material, which is available to authorized users.

Ilse Dewachter

ilse.dewachter@uclouvain.be

1 Alzheimer Dementia Group, Institute of Neuroscience, Catholic University of Louvain, 1200 Brussels, Belgium

2 Department of Neuroscience, Janssen Research and Development, A Division of Janssen Pharmaceutica NV, 2340 Beerse, Belgium

3 VIB11 vzw Center for the Biology of Disease, KU Leuven, 3000 Leuven, Belgium induction and propagation of Tau-pathology in vivo. Prionlike, heterotypic seeding of Tau fibrillization by $\mathrm{A} \beta$, providing potent seeds for propagating Tau pathology in vivo, as demonstrated here, provides a compelling molecular mechanism for $\mathrm{A} \beta$-induced propagation of Tau-pathology, beyond regions with pre-existing Tau-pathology (entorhinal cortex/locus coeruleus). Cross-seeding along functional connections could thereby resolve the initial spatial dissociation between amyloid- and Tau-pathology, and preferential propagation of Tau-pathology in regions with pre-existing 'silent' Tau-pathology, by conversion of a 'silent' Tau pathology to a 'spreading' Tau-pathology, observed in AD.

Keywords Amyloid beta $\cdot$ Tau $\cdot$ Heterotypic seeding · Prion-like $\cdot$ Alzheimer's disease

\section{Introduction}

Brains of $A D$ patients are characterized by the presence of neurofibrillary tangles and amyloid plaques composed of aggregated Tau and $\mathrm{A} \beta$ peptides, respectively [46, 47]. In $\mathrm{AD}$, spreading of Tau-aggregation occurs in a very characteristic pattern (Braak stages) along functionally connected brain areas [3], strongly correlating with symptom progression, explaining its use as diagnostic criteria. Combined genetic, biomarker and histopathological data indicate increasing $A \beta$ peptide concentrations as driving force for the conversion from preclinical to mild cognitive impairment (MCI) and finally AD [19, 25, 26, 53], strongly correlating with spreading of Tau-pathology beyond EC and the limbic system to the neocortex, by a poorly understood molecular mechanism. Noteworthy, a spatial dissociation between predominant amyloid- and Tau-pathology exists in (the initial stages of) AD. Amyloid-pathology accumulates 
predominantly in isocortex (Thal stages) [54], while Taupathology (Braak stages) [3] spreads from EC in a characteristic pattern along functionally connected regions.

While mechanistically unclear, $A \beta$-induced Tau-pathology has been demonstrated reproducibly in in vitro and in vivo models $[2,5,13,29,36,40,49,50]$. Indeed, in a human neural stem cell derived 3D culture system, expression of EOFAD mutations induced aggregated $A \beta$ and Tau filaments, with Tauopathy downstream of $A \beta$ formation [5]. Furthermore, different milestone papers have demonstrated amyloid-induced Tau-pathology in in vivo animal models, including following (1) crossing or co-expressing of mutant APP/PS1 with mutant Tau in transgenic mice [2, 29, 36, 40, 49] following (2) injection of pre-aggregated $A \beta$ containing extracts derived from AD patients or APP/PS1 transgenic mice [2], or (3) following injection of synthetic pre-aggregated $A \beta$ [13]. Intriguingly, $A \beta$-induced Tau-pathology, following injection of pre-aggregated $A \beta$, induced not only Tau-pathology at the site of injection but also in brain regions remote from, but functionally connected to the injection site $[2,13]$.

These findings are not only reminiscent of the spatial dissociation between amyloid and Tau-pathology in the initial stages in $\mathrm{AD}$ patients, but also reminiscent of the prionlike spreading of Tau-pathology following Tau-seeding, along functional connections $[1,6,22,51]$. Prion-like properties have been demonstrated for different proteins associated with neurodegenerative disorders $[6,8,12,16,17$, 22, 27, 28, 30, 33, 38, 39, 51, 56], including Tau. Prion-like seeding and propagation of Tau-pathology have been demonstrated in vitro and in vivo in different models with synthetic or patient-derived pre-aggregated Tau $[6,16,17,22$, $28,39,44,51]$. Prion-like "seeding" using pre-aggregated Tau fibrils propagates Tau-aggregation by overcoming the rate-limiting nucleation step of Tau-aggregation. Once initiated Tau-pathology propagates rapidly in a prion-like way in in vivo models, and according to a pattern observed in $\mathrm{AD}$, following initiation in EC Tau-pathology spreads to limbic system and cortex [7, 22, 27, 51]. Tau-pathology is thereby propagated to remote but functionally connected brain regions, affecting intrinsic functional neuronal networks, reminiscent of the networks affected in $\mathrm{AD}$ and related Tauopathies $[1,22,51]$. Although mechanistically not yet fully resolved, in terms of uptake and spreading of "seeds", this process has been reproducibly recapitulated in different models. The strong similarity in induction of Tau-pathology by prion-like Tau-seeds and pre-aggregated forms of $A \beta$ in in vivo models (i.e. in crosses of transgenic mice, following injection of pre-aggregated $A \beta$ containing brain extracts from mice and $\mathrm{AD}$ patients, and preaggregated synthetic $A \beta$ ) has incited us to analyze whether pre-aggregated $A \beta$ could directly cross-seed filamentous Tau-aggregation, leading to propagation of Tau pathology.
Interestingly, cross-seeding between Tau and alpha-synuclein has been demonstrated previously in vitro and in vivo $[11,15]$. Hence, in this work we have analyzed the potential of $A \beta$-seeds to induce Tau-aggregation and the potential of these $A \beta$-induced Tau-seeds to seed propagating Taupathology in vivo, as a potential mechanism underlying the propagation of Tau-pathology beyond $\mathrm{EC}$ in $\mathrm{AD}$.

\section{Materials and methods}

\section{In vitro generation of monomeric and pre-aggregated seeds}

In vitro generation of pre-aggregated synthetic A $\beta \quad 1-42$ peptides $\mathrm{A} \beta$ 1-42 peptides were purchased from Bachem (Bachem AG, Switzerland). $A \beta$ monomers and $A \beta$ aggregates (fibrils) were prepared as previously described [52]. Briefly, lyophilized monomeric A $\beta$ 1-42 was resuspended in HFIP (Sigma-Aldrich) and subsequently evaporated for $1 \mathrm{~h}$ in a SpeedVac (Thermo Fisher Scientific, Waltham, MA, USA) before its storage in single use aliquots at $-20{ }^{\circ} \mathrm{C}$. For $\mathrm{A} \beta \quad 1-42$ aggregation, the stored monomeric A $\beta$ 1-42 was resuspended in DMSO (Sigma-Aldrich) at $5 \mathrm{mM}$, before dilution to $100 \mu \mathrm{M}$ in $10 \mathrm{mM} \mathrm{HCl}$ solution and incubation $24 \mathrm{~h}$ at $37^{\circ} \mathrm{C}$. The aggregation nature of the preparations was assessed by thioflavin $\mathrm{T}$ (ThioT) assay, immunoblotting and immuno-EM. For all the experiments only freshly prepared sonicated fibrils (8 pulses of $30 \%$ amplitude) were used.

In vitro generation of monomeric and pre-aggregated Tau- "Tau seeds" Tau seeds were generated as previously described [16, 22, 51]. The human truncated 4R Tau, encompassing the 4-repeat microtubule binding domain of Tau with the P301L mutation and a myc tag (K18-P301L; Q244-E372) was generated in Escherichia coli, further referred to as Tau ${ }^{\text {Mono }}$. Pre-aggregated synthetic Tau, "Tau seeds", were obtained by incubation of Tau ${ }^{\text {Mono }}(66.7 \mu \mathrm{M})$ with low molecular weight heparin [MP Biomedicals, Santa Ana, CA, USA; (ratio 1:2)] in $100 \mathrm{mM}$ ammonium acetate buffer ( $\mathrm{pH} 7$ ) at $37{ }^{\circ} \mathrm{C}$ for 5 days. Before use, fibrillization mixture was centrifuged $\left(100,000 \mathrm{~g}\right.$ for $1 \mathrm{~h}$ at $\left.4{ }^{\circ} \mathrm{C}\right)$ and the resultant pellet resuspended in the same buffer without heparin to a final concentration of $333 \mu \mathrm{M}$ and stored at $-80^{\circ} \mathrm{C}$. Successful Tau fibrillization was confirmed by ThioT (Sigma-Aldrich, St. Louis, MO, USA) assay, immunoblotting and immuno-EM. For all experiments Tau seeds were sonicated ( 8 pulses of $30 \%$ amplitude) before use.

In vitro generation of pre-aggregated synthetic amylin peptides Amylin peptides were purchased from Bachem (Bachem AG, Switzerland). Amylin aggregates were prepared as described [42, 43]. Briefly, amylin peptides were dissolved in DMSO to a final concentration of $20 \mathrm{mM}$ 
and stored at $-20{ }^{\circ} \mathrm{C}$. For amylin aggregation, the stored monomeric peptides were dissolved in $25 \mu \mathrm{M} \mathrm{KCl}$ to a final concentration of $20 \mu \mathrm{M}$ and incubated at $37{ }^{\circ} \mathrm{C}$ for $65 \mathrm{~h}$. The aggregation nature of the preparations was assessed by ThioT assay and immunoblotting. For all the experiments only freshly prepared sonicated fibrils (8 pulses of $30 \%$ amplitude) were used.

\section{Cell culture and Tau aggregation assay}

Human kidney-derived QBI-293 (QBiogene, Carlsbad, CA, USA) were grown in Dulbecco's modified Eagle's medium (DMEM) supplemented with $10 \%(\mathrm{v} / \mathrm{v})$ heat inactivated FBS, $1 \%$ Pyruvate $(10 \mathrm{mM}), 1 \%$ Penicillin-Streptomycin (PenStrep) and L-glutamine $(20 \mathrm{mM})$. Cells were maintained at $37{ }^{\circ} \mathrm{C}$ in humidified atmosphere containing $5 \% \mathrm{CO}_{2}$. One day prior to transfection, $80 \%$ confluent cells were trypsinized and then seeded in $10 \mathrm{~cm}^{2}$ dishes at $1.5 \times 10^{6}$ cells per well. The growth medium was renewed directly before transfection. DNA mixture containing $2.5 \mu \mathrm{g}$ pcDNA6-TR and $2.5 \mu \mathrm{g}$ 2N4R-TauP301LGFP-pcDNA4/TO was diluted in $500 \mu \mathrm{L}$ OptiMEM and $15 \mu \mathrm{L} \mathrm{FuGENE}{ }^{\circledR} 6$ transfection reagent diluted in $500 \mu \mathrm{L}$ OptiMEM was added. The mixture was incubated for $15 \mathrm{~min}$ at room temperature (RT), then added to the cells. After incubation for $24 \mathrm{~h}$, the growth medium was removed and replaced with a new one containing $5 \mu \mathrm{g} / \mathrm{mL}$ blasticidin and $200 \mu \mathrm{g} / \mathrm{mL}$ Zeozin. The cells were cultured until selection was complete. Monoclonal lines were generated by limited dilution. Cells were then grown in full media (DMEM, 10 \% FBS; Invitrogen, Life Technologies, Carlsbad, CA, USA) supplemented with penicillin/streptomycin, L-glutamine and pyruvate. For the Tau aggregation assays, cells were plated at 100,000 per well in 12-well plates in medium containing doxycycline (Westburg, Leusden, Netherlands). On the day of aggregates transfection, Tau-seeds and $\mathrm{A} \beta$-seeds were diluted in the respective buffers to a $10 \mu \mathrm{M}$ solution and sonicated with 8 pulses $/ 30 \%$ amplitude; $80 \mu \mathrm{L}$ of this solution was added to single use BioPORTER tubes (AMS Biotechnology, Milton, UK), vortexed at low speed for $5 \mathrm{~s}$ and incubated $10 \mathrm{~min}$ at RT. During the seeds-BioPORTER complexes formation, cells were washed with OptiMEM (Invitrogen) and placed in $250 \mu \mathrm{L}$ of OptiMEM. $420 \mu \mathrm{L}$ of OptiMEM was added to the BioPORTER tubes, and $250 \mu \mathrm{L}$ of the resultant solution was added to the cells. The cells were incubated with the seeds for $4 \mathrm{~h}$, and subsequently $500 \mu \mathrm{L}$ medium was added (complete medium containing $20 \%$ FBS) and further incubated for 3 days. For experiments using 24-well plates, the volumes were adjusted accordingly. For the experiments using $A \beta$ seeds as catalyst of pre-existing Tau aggregation, two "BioPORTER transfections" were performed. On the first day of the experiment,
Tau-seeds were transfected to the cells followed by the transfection of $\mathrm{A} \beta$-seeds $24 \mathrm{~h}$ later. For the control condition BioPORTER without $A \beta$-seeds was added. The cells were further incubated for 2 days.

\section{Biochemical analysis}

For total cell extracts, cells were washed and scraped in OptiMEM and centrifuged at $1000 \mathrm{~g}$ for $5 \mathrm{~min}$ at $4{ }^{\circ} \mathrm{C}$. Cell pellets were then resuspended in Triton lysis buffer $(1 \%$ TX-100, $50 \mathrm{mM}$ Tris, $150 \mathrm{mM} \mathrm{NaCl}, \mathrm{pH}$ 7.6) containing proteases and phosphatases inhibitors (F. Hoffman-La Roche AG, Basel, $\mathrm{CH}$ ) before storage at $-20^{\circ} \mathrm{C}$. For the separation of soluble and insoluble Tau, the Triton/SDS method was used as previously described [16, 22, 51]. Briefly, cells were washed with PBS, scraped into Triton lysis buffer containing protease and phosphatase inhibitors and incubated on ice for $15 \mathrm{~min}$. Cell extracts were sonicated and centrifuged at $100,000 \mathrm{~g}$ for $30 \mathrm{~min}$ at $4{ }^{\circ} \mathrm{C}$. The supernatants yielded the Triton-soluble fraction (soluble proteins), while the pellets were resuspended, sonicated and vortexed in SDS lysis buffer (1\% SDS, $50 \mathrm{mM}$ Tris, $150 \mathrm{mM} \mathrm{NaCl}, \mathrm{pH}$ 7.6) and stored as Triton-insoluble fraction, after centrifugation at $100,000 \mathrm{~g}$ for $30 \mathrm{~min}$ at $28^{\circ} \mathrm{C}$. For all samples proteins were quantified using BCA Protein Assay kit (Thermo Fisher Scientific, Waltham, MA, USA). Samples were run in precast 4-12\% Bis-Tris gels (MOPS running; Invitrogen) and immunoblotting was performed with anti-Tau P-S202/T205 (AT8 1:500; Thermo Fisher Scientific), anti-total Tau antibody (HT7 1:500; Thermo Fisher Scientific) and anti-actin antibody (1:500; SigmaAldrich) and developed using ECL kit (PerkinElmer, Waltham, MA, USA).

\section{Cytological and immunocytological analysis}

Cells were fixed under stringent conditions for the removal of soluble Tau proteins using fixation/extraction buffer (4\% PFA, $4 \%$ sucrose, $1 \%$ TX-100 in PBS) for 10 min. After washing with PBS, cells were blocked (5\% FCS or milk in $0.1 \% \mathrm{TX}-100 \mathrm{PBS}$ ) for $30 \mathrm{~min}$. The specific anti-Tau P-S202/T205 (AT8 1:100) and anti-conformational specific Tau (MC1 1:100, Peter Davies) were incubated for $2 \mathrm{~h}$ at RT or overnight at $4{ }^{\circ} \mathrm{C}$ followed by staining with Alexa Fluor 568 goat anti-Mouse IgG1 (1:500) or Alexa Fluor 647 goat anti-Mouse IgG1 (1:500) for $1 \mathrm{~h}$ at RT. DAPI staining to label nuclei was performed in a PBS washing step. The GFP bleaching experiments were performed as previously described with minor modifications [32]. Briefly, a $5 \mu \mathrm{M}$ acid-denatured GFP solution ( $\mathrm{pH} 1.5$ ) was prepared by mixing equal amounts of solution $\mathrm{A}(50 \mathrm{mM}$ Tris- $\mathrm{HCl}$ $\mathrm{pH}$ 7.5) with solution $\mathrm{B}(125 \mathrm{mM} \mathrm{HCl})$ and incubated with cells 1 min at RT. Image acquisition was performed using 
a digital inverted fluorescence microscope (EVOS-xl auto microscope). The $10 \times, 20 \times$ and $40 \times$ coverslip corrected lens were used in this study.

\section{In vitro Tau aggregation assay (cell-free assay)}

To assess the ability of $A \beta$ seeds to directly cross-seed Tau aggregation, in vitro Tau fibrillization experiments were performed. Tau ${ }^{\text {Mono }}(66.7 \mu \mathrm{M})$ was incubated with heparin $(133 \mu \mathrm{M})$, pre-aggregated synthetic Tau seeds (6.67 $\mu \mathrm{M}$; buffer $\mathrm{A}), \mathrm{A} \beta$ seeds $(6.67 \mu \mathrm{M}$; buffer $\mathrm{B})$, amylin seeds $(6.67 \mu \mathrm{M})$, albumin $(6.67 \mu \mathrm{M})$, or with buffers alone (diluted as used in the respective seeding conditions). The fibrillization reaction was performed for 3 days at $37{ }^{\circ} \mathrm{C}$ with constant agitation at $150 \mathrm{rpm}$. Aliquots of all reactions were collected at different time points and successful fibrillization of Tau was verified by ThioT assay, sedimentation test and immunogold electron microscopy.

\section{ThioT assay}

The assay was performed as previously described [10, 55]. Briefly, samples were incubated with ThioT solution ( $5 \mu \mathrm{M}$ ThioT in $100 \mathrm{mM}$ ammonium acetate buffer $\mathrm{pH}$ 7.0) in 96-well plates at RT. Fluorescence was measured with a PerkinElmer Victor X3 (PerkinElmer) with an excitation filter of $440 \mathrm{~nm}$ and an emission filter of $486 \mathrm{~nm}$.

\section{Sedimentation analysis}

The sedimentation analysis was performed as previously described with minor modifications [11]. Briefly, samples were ultracentrifuged $\left(100,000 \mathrm{~g}\right.$ for $1 \mathrm{~h}$ at $\left.4{ }^{\circ} \mathrm{C}\right)$ and the resultant pellet was resuspended in a volume of buffer corresponding to the volume of supernatant. Western Blot analysis was performed using an anti-total Tau antibody (1:2000; DAKO, Glostrup, Denmark).

\section{Immunogold electron microscopy}

Immunogold labeling was performed as previously described [51]. Pure protein samples of the in vitro Tau fibrillization experiments were applied on 200-mesh carbon-coated grids in drops of $2 \mu \mathrm{L}$ for $5 \mathrm{~min}$, blotted on filter paper and air-dried before immunogold labeling. Grids were blocked in PBS $0.1 \%$ cold water fish gelatin (PBSCWFG) for 5 min before incubation with PBS-CWFG diluted primary antibody (Polyclonal Total Human Tau 1:50) for $90 \mathrm{~min}$ at RT. After washing five times $(2 \mathrm{~min})$ in PBS-CWFG, grids were incubated for $30 \mathrm{~min}$ with protein A secondary antibody labeled with $10 \mathrm{~nm}$ gold particles (Aurion, Wageningen, Netherlands) diluted 1:50 in PBSCWFG. Following washes in PBS and ddH2O, grids were
Fig. 1 Pre-aggregated $A \beta$-seeds induce Tau-aggregation in a wellcharacterized in vitro Tau-aggregation assay. a Schematic design of seed induced Tau-aggregation in QBI-293 cells expressing TauP301L-GFP. Pre-aggregated Tau-seeds or A $\beta$-seeds are added to the cells thereby bypassing the slow nucleation-dependent lag phase. Following stringent extraction using $1 \%$ TX-100 during PFA fixation, soluble forms of Tau are eliminated. The remaining GFP signal represents aggregated GFP-tagged TauP301L. b Similar to Tau-seeds, $\mathrm{A} \beta$-seeds induce Tau-aggregation in the in vitro Tau-aggregation assay, albeit less efficiently than Tau-seeds (scale bar $400 \mu \mathrm{m}$ ). Quantitative analysis reveals significant $\mathrm{A} \beta$-seed induced Tau aggregation ( $n \geq 30$ fields per condition; Kruskal-Wallis test followed by Dunn's multiple comparison post hoc test, indicating significant difference compared to non-seeded condition; mean \pm SEM are presented; $* * p$ value $<0.01, * * * p$ value $<0.001)$. c Higher magnification images of the different conditions are presented. Aggregated Tau is stained with $\mathrm{MC1}$ antibody, detecting conformationally altered Tau, further confirming the seed induced Tau pathology, absent in non-seeded cells, and not significantly induced in cells treated with monomeric $\mathrm{A} \beta$ forms or monomeric Tau forms (Fig. S2) (scale bar $100 \mu \mathrm{m}$ )

negatively stained with $2 \%$ uranyl acetate for $45 \mathrm{~s}$. Grids were examined with a JEOL JEM1400 transmission electron microscopy equipped with an Olympus SIS Quemesa $11 \mathrm{Mpxl}$ camera, and images were taken at magnifications of $10 \times, 20 \times$ and $30 \times \mathrm{k}$. For co-staining, grids were sequentially incubated for $90 \mathrm{~min}$ with the primary mouse anti-A $\beta$ antibody (WO2 1:50) followed by rabbit anti-total human Tau antibody (DAKO Tau 1:50). Subsequently the grids were incubated with secondary antibodies at RT in PBS-CWFG. The secondary antibodies used were, respectively, $10 \mathrm{~nm}$ gold coupled goat anti-mouse antibody (1:50 in PBS-CWFG) and $5 \mathrm{~nm}$ gold coupled goat anti-rabbit antibody (1:50 in PBS-CWFG). The grids were negatively stained with $2 \%$ uranyl acetate for $45 \mathrm{~s}$ and were examined as previously described.

\section{In vivo seeding experiments}

Animals TauP301S mice (PS19) [58] expressing the Tau isoform with one $\mathrm{N}$-terminal insert and four microtubule binding repeats $(1 \mathrm{~N} 4 \mathrm{R})$ with the P301S clinical mutation, driven by the prion protein promotor, were backcrossed with C57B6 mice and used in this study. The TauP301S mice used in our lab develop similar neuropathology to previously reported studies, at around 11 months [22, 50, 58]. Animals were housed on a $12 \mathrm{~h}$ light/dark cycle in specific pathogen-free (SPF) facilities with access to food and water ad libitum. Stereotactic injections were performed in 4 months old mice and age-matched littermates were used for analysis at 3 months post-injection. All experiments were performed in compliance with protocols approved by the UCLouvain Ethical Committee for Animal Welfare.

Stereotactic injections For stereotactic surgery, 4 months old mice were deeply anesthetized with a mixture of ketamine/xylazine (Ketalar/Rompun). Unilateral (right 
a $A \beta$ induced Tau-aggregation

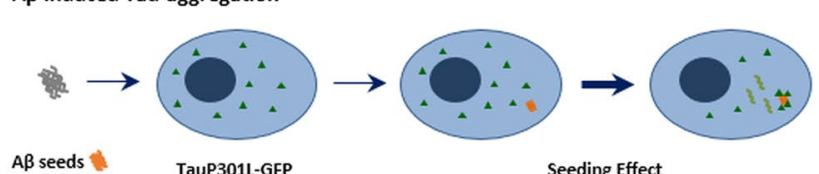

Tau seeds

b
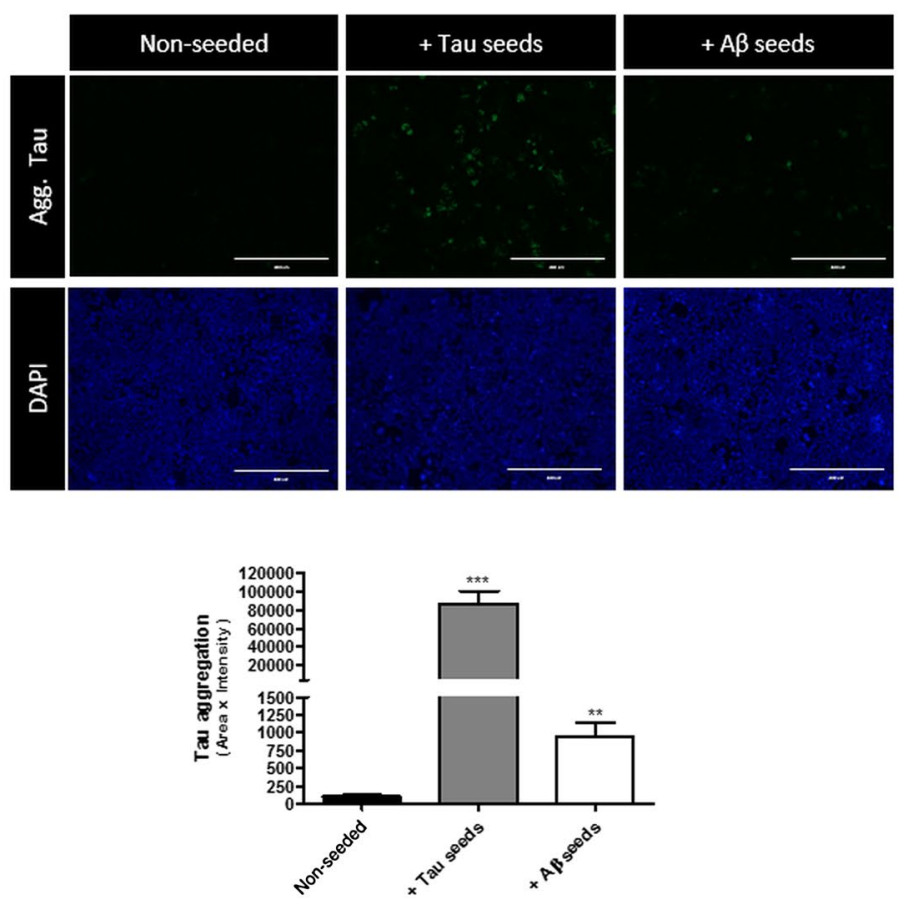

c
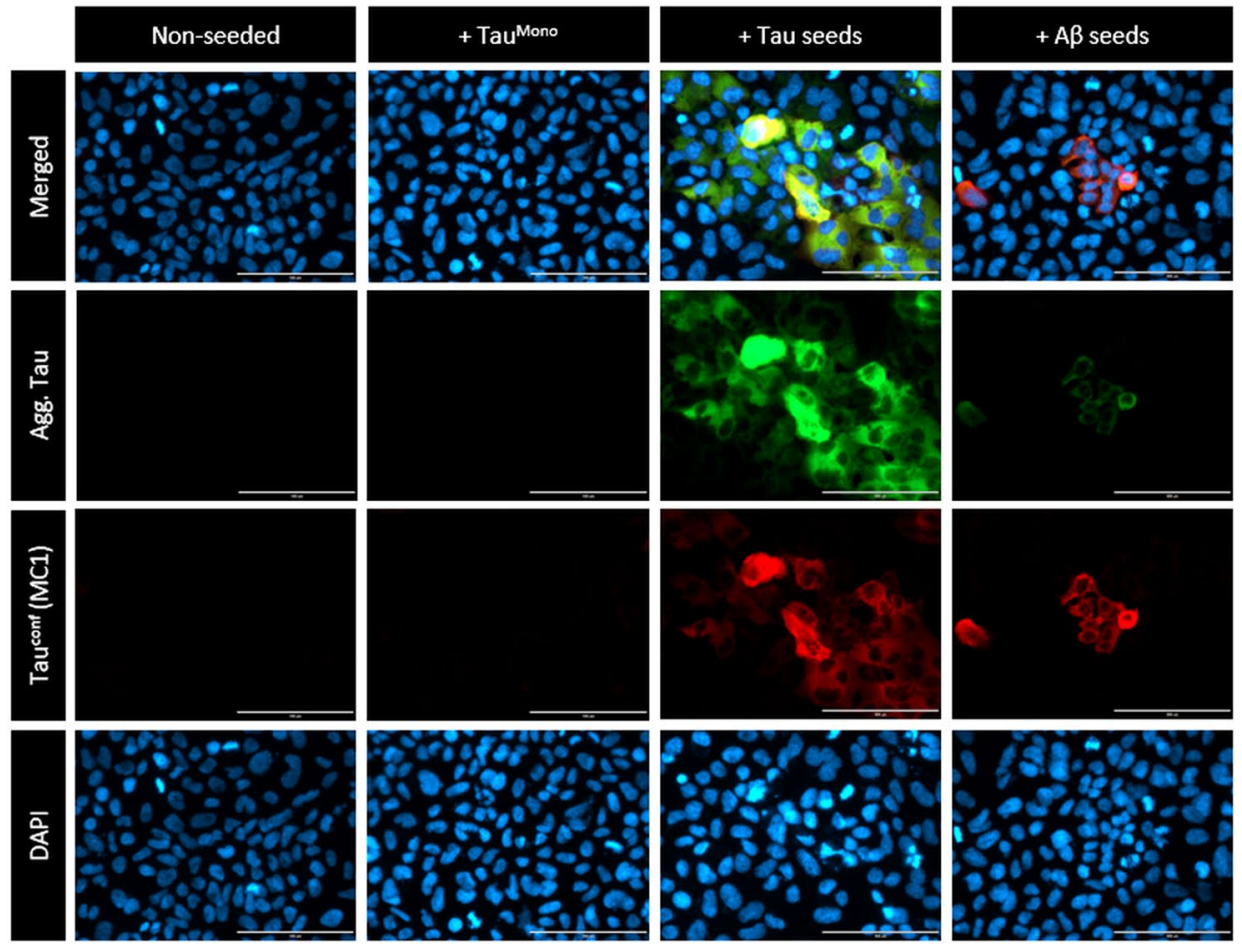
hemisphere) stereotactic injections were performed in the hippocampal region $(\mathrm{A} / \mathrm{P},-2.0 \mathrm{~mm} ; \mathrm{L},+1.4 \mathrm{~mm} ; \mathrm{D} / \mathrm{V}$, $-1.4 \mathrm{~mm})$ and frontal cortex $(\mathrm{A} / \mathrm{P},+2.0 \mathrm{~mm}$;,$+1.4 \mathrm{~mm}$; $\mathrm{D} / \mathrm{V},-1.0 \mathrm{~mm}$ ); all coordinates are expressed relative to bregma [37]. Sonicated pre-aggregated homotypic Tauinduced Tau-aggregates $(66.7 \mu \mathrm{M}), \mathrm{A} \beta$-induced Tauaggregates $(66.7 \mu \mathrm{M})$, non-aggregated or monomeric Tau controls $(66.7 \mu \mathrm{M}), \mathrm{A} \beta$ seeds $(333 \mu \mathrm{M})$ and buffer controls were injected using a $10 \mu \mathrm{L}$ Hamilton syringe at a speed of $1 \mu \mathrm{L}$ per min. After injection, the needle was kept in place for additional $5 \mathrm{~min}$ before gentle withdrawal.

Immunohistological analysis Immunohistological analysis was performed as previously described [41, 50]. Animals were transcardial perfused with ice cold PBS $(2 \mathrm{~min})$ by transcardial flushing and brains were dissected and fixed in $4 \%$ PFA in PBS for $24 \mathrm{~h}$ at $4{ }^{\circ} \mathrm{C} .40 \mu \mathrm{m}$ thick sagittal sections were cut in a vibrating HM650V microtome (Thermo Fisher Scientific, Waltham, MA, USA). Immunohistochemistry (IHC) was performed on these sections with anti-Tau P-S202/T205 (AT8), anti-Tau P-S212/T214 (AT100) and anti-conformational Tau (MC1, Peter Davies) primary antibodies followed by appropriate Alexa-coupled secondary antibody, as previously described [50]. Staining with Thioflavin S (ThioS; Sigma-Aldrich, St. Louis, MO, USA) and Gallyas silver (all chemicals from Sigma-Aldrich, St. Louis, MO, USA) staining were performed as previously described $[50,51]$ and were used to demonstrate the "amyloid" nature of Tau aggregates in brain sections. Image acquisition was performed using a digital inverted fluorescence microscope (EVOS-xl auto microscope), using the $4 \times, 10 \times$ and $20 \times$ objectives. Image analysis was performed using Image $\mathbf{J}$ (National Institutes of Health). Heat maps were generated using the HeatMap Histogram plugin for Image $\mathrm{J}$, as previously described [51]. Briefly, the overview images of AT8 staining of a well-identified section of different mice $(n=6$ per group) were grouped. Stacked images representing averaged intensities were generated using the Image $\mathbf{J}$ stacking tool with the average intensities outcome option. Finally a Gaussian Blur filter of 5.0 was applied.

\section{Statistical analysis}

Statistical analysis was performed using GraphPad Prism 5.0 (GraphPad Software, San Diego, CA, USA). Normality testing was performed for all the data using D'Agostino and Pearson omnibus normality test when possible. Kolmogorov-Smirnov tests were used for smaller sample sizes. According to the results of normality tests appropriate statistical tests were used, including one-way analysis of variance (ANOVA) followed by Tukey's post hoc test, two-way ANOVA followed by Bonferroni post hoc test and Kruskal-Wallis test followed by Dunn's multiple comparison post hoc test. The tests used are indicated in the legends
Fig. 2 Pre-aggregated $A \beta$-seeds strongly accelerate incipient or preexisting Tau-aggregation. a Schematic presentation of the assay. Following induction of Tau-aggregation with Tau-seeds in TauP301LGFP expressing cells, cells are subsequently seeded with $A \beta$-seeds or control conditions. Analysis is performed by cytological analysis following stringent extraction to eliminate soluble forms of Tau. b Seeding with Tau-seeds induced Tau-aggregation as demonstrated previously, however, subsequent seeding with $\mathrm{A} \beta$-seeds strongly catalyzes Tau-aggregation compared to control conditions ("mock") (scale bar $400 \mu \mathrm{m}$ ). Quantitative analysis of Tau-aggregation demonstrates significantly increased Tau-aggregation following subsequent seeding with $\mathrm{A} \beta$-seeds ( $n \geq 36$ fields per condition; Kruskal-Wallis test followed by Dunn's multiple comparison post hoc test, indicating significant difference in all conditions compared to non-seeded condition, and significant difference between sequential seeding with $\mathrm{A} \beta$ and single Tau seeding; mean \pm SEM are presented; $* * p$ value $<0.01$, $* * * p$ value $<0.001)$. c Higher magnifications of the different experimental conditions are provided. Aggregated Tau is further confirmed to display conformational alterations by staining with $\mathrm{MC} 1$ antibody (scale bar $100 \mu \mathrm{m}$ )

of the figures. Data were expressed as mean \pm SEM and differences were considered significant when $p$ values $<0.05$.

\section{Results}

\section{Pre-aggregated $\mathrm{A} \beta$-seeds induce Tau-aggregation in a well-characterized cellular Tau aggregation assay}

To start exploring molecular mechanisms of $\mathrm{A} \beta$-induced Tau-aggregation we used a previously described and characterized cellular Tau-aggregation assay [16, 44, 51]. In this assay, the nucleation-dependent long initial lag phase for Tau-fibrillization in cells is by-passed by the addition of preformed pre-aggregated synthetic Tau-seeds, which accelerate fibrillization of monomeric Tau. Hereto, QBI293 cells were transfected with the longest human Tau isoform (2N4R) with the P301L mutation, to optimize Tau aggregation, and transduced with preformed fibrils generated from Myc-tagged truncated Tau P301L containing only the four MT-binding repeats-further referred to as Tau-seeds when aggregated and $\mathrm{Tau}^{\mathrm{Mono}}$ in their monomeric form-using BioPORTER protein delivery reagent. GFP-tagged Tau displayed similar aggregation as nontagged Tau, and was therefore used in the assay to facilitate detection of Tau-aggregation. Cells transduced with Tauseeds displayed accumulation of Triton-insoluble phospho-Tau aggregates as demonstrated by Western blotting analysis, while no Tau-aggregates were detected following transduction with BioPORTER only (non-seeded) (Fig. S1). For cytological analysis, a stringent extraction was performed during fixation (using $1 \% \mathrm{TX}-100$ ) to eliminate soluble Tau forms, leaving aggregated Tau detectable following Tau-seeding with Tau-seeds. These aggregates 
a $A \beta$ acceleration of Tau-aggregation

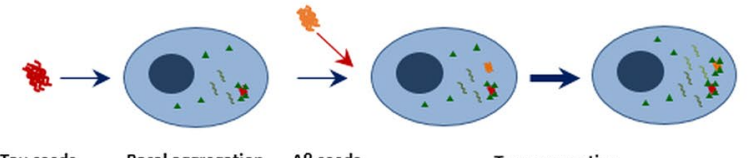

Tau seeds
Tau aggregation

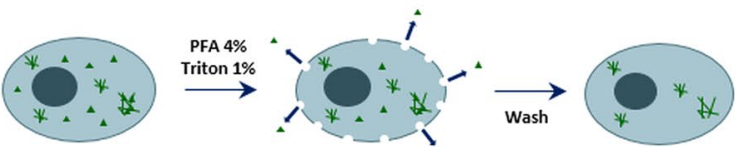

Green signal = Tau aggregation

b
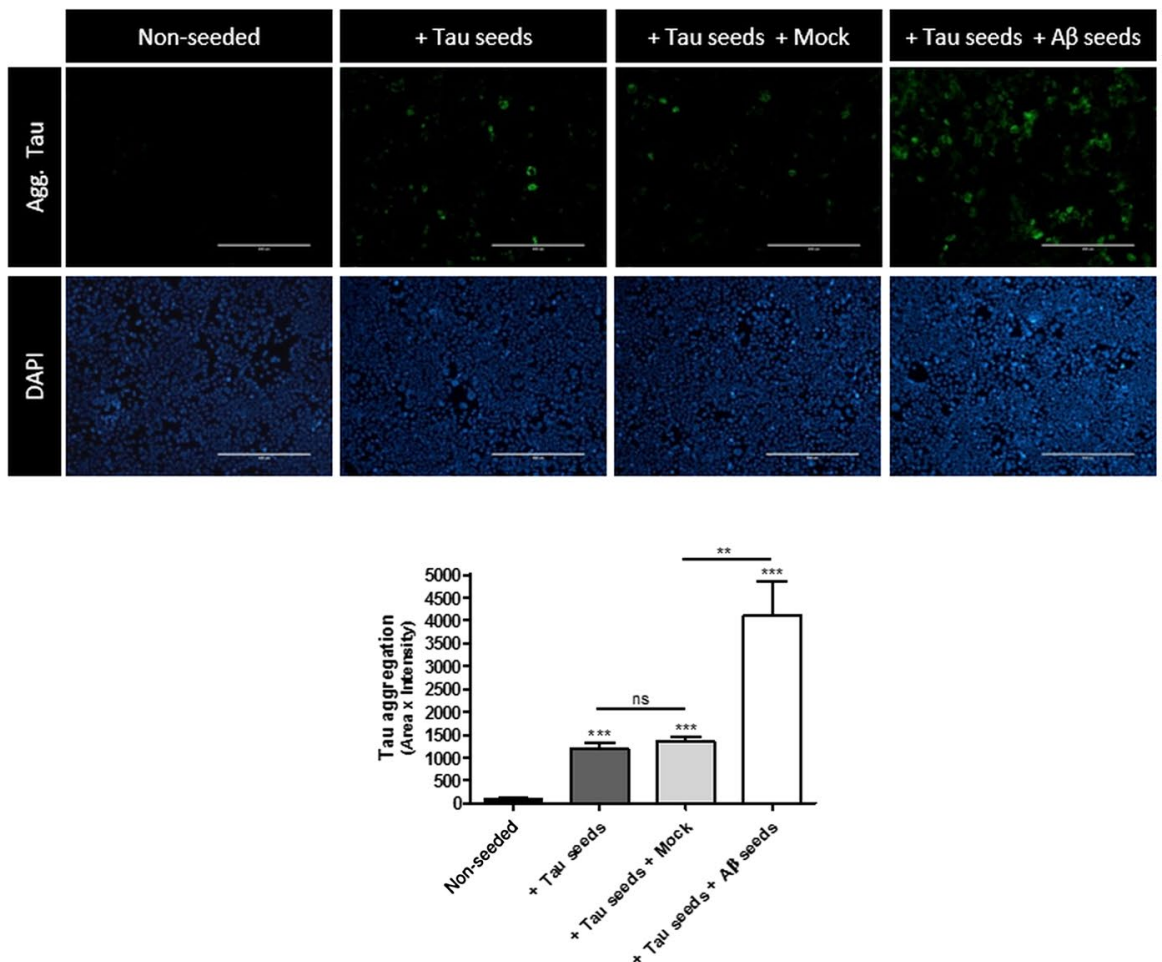

c
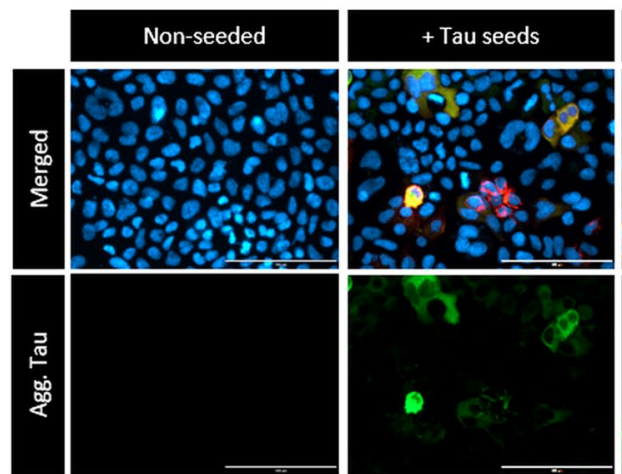

+ Tau seeds + Mock

+ Tau seeds $+A \beta$ seeds
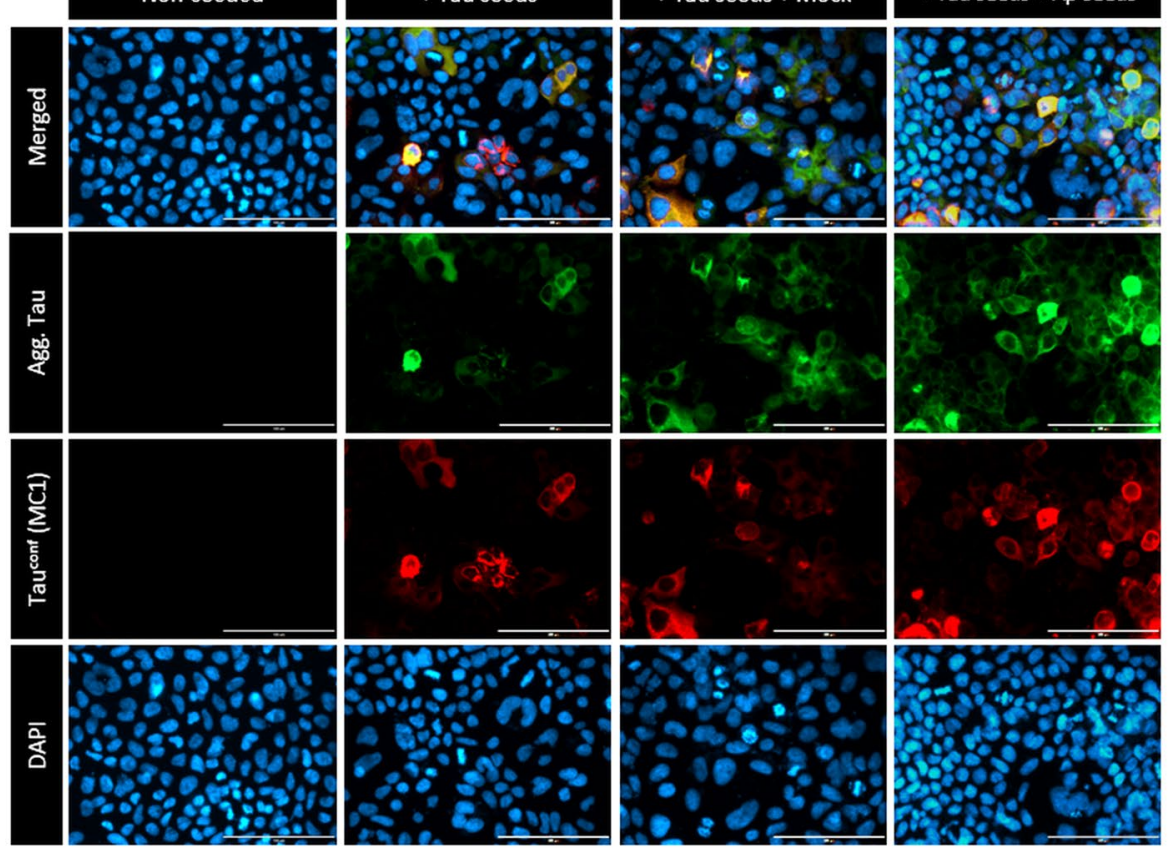
were also detected by anti-phospho-Tau primary antibody AT8 (S202/T205) and conformation-dependent anti-Tau antibody MC1 (Fig. S1). This assay is well characterized, has been extended to different cell types, is reproducible in different labs and has previously been used to identify the existence of different Tau prion-strains [16, 22, 44, 51]. It was also used to evaluate the potential of cross-seeding of Tau and alpha-synuclein in cells $[15,44,57]$. Here, we used this assay to analyze whether pre-aggregated $A \beta$ seeds could facilitate Tau-aggregation, in a similar way as pre-aggregated Tau-seeds. We used this Tau-aggregation assay for analysis of seeding with pre-aggregated synthetic Tau-seeds, pre-aggregated synthetic $\mathrm{A} \beta$ (A $\beta$ 1-42) fragments or BioPORTER alone (Fig. 1). Seeding with pre-aggregated synthetic Tau fragments significantly and robustly induced Tau-aggregation as demonstrated by the presence of Tau-GFP signal following stringent extraction with $1 \%$ TX-100, validating the assay. Strikingly, seeding with pre-aggregated $A \beta$, further referred to as " $A \beta$-seeds", induced Tau-aggregation in a reproducible consistent way (Fig. 1). In contrast to pre-aggregated $A \beta$-seeds and preaggregated Tau-seeds, their monomeric forms (monomeric Tau and monomeric $\mathrm{A} \beta$ ) did not induce significant Tauaggregation in this assay (Fig. S2). Notably, Tau-aggregation as a consequence of pre-aggregated $\mathrm{A} \beta$ cross-seeding was markedly less strong than Tau-aggregation induced with pre-aggregated Tau-fragments, but was consistently induced in each well in each experiment. This was further confirmed by quantitative analysis demonstrating significantly increased induction of Tau-pathology by $A \beta$-seeds (Fig. 1).

\section{Pre-aggregated A $\beta$-seeds accelerate incipient Tau-aggregation in a cellular Tau aggregation assay}

In view of the fact that Tau-aggregation can be present in locus coeruleus (LC) and entorhinal cortex (EC) already early in life, but only spreads from EC to functionally connected brain regions during conversion to $\mathrm{MCI}$ and $\mathrm{AD}-$ generally hypothesized due to increasing $A \beta$ burden-, we analyzed whether pre-aggregated $A \beta$ could accelerate propagation of incipient Tau-pathology [3, 19, 31]. Hence, we analyzed whether $A \beta$-seeds could aggravate incipient Taupathology in this cellular Tau-aggregation assay (Fig. 2). Hereto, Tau-seed induced Tau aggregation was followed by an additional seeding with $\mathrm{A} \beta$-seeds. As demonstrated previously, Tau-seeding induced robust Tau-aggregation, however, quantitation of the aggregated Tau by IF revealed a striking increase of Tau-aggregation following additional seeding with $\mathrm{A} \beta$ (Fig. 2). Taken together, our data in the cellular Tau-aggregation assay indicate that $A \beta$-seeds can directly seed Tau-aggregation and can strongly accelerate propagation of pre-existing Tau-aggregates. The latter data are relevant in the context of spreading of pre-existing Taupathology from entorhinal cortex to functionally connected brain regions, driven by an increasing $\beta$-amyloid load. Preaggregated $\mathrm{A} \beta$ may thereby act as seeds to bypass or accelerate the lag-phase of Tau-aggregation by providing nucleation templates for Tau-aggregation.

\section{Pre-aggregated A $\beta$ induces Tau fibrillization, in a cell-free assay, providing direct proof of heterotypic seeding}

To further explore the hypothesis of direct induction of Tau-fibrillization by cross-seeding with pre-aggregated $\mathrm{A} \beta$ we used a cell-free assay similarly as previously used for demonstrating cross-seeding of alpha-synuclein and Tau [11]. We assessed if $A \beta$ could directly cross-seed Tau-aggregation in a biochemical, cell-free assay (Fig. 3). Direct heterotypic seeding between pre-aggregated $A \beta$ and Tau would be supported by previous data, demonstrating direct binding between $A \beta$ peptides and Tau [18] and in silico models for $A \beta$-induced beta-sheet formation of Taufragments [34]. However, the final proof, i.e. $A \beta$-induced Tau fibril formation and its subsequent propagating potential, has not been demonstrated. In this biochemical assay Tau fibrillization is dependent on a rate-limiting nucleation-phase, which has been demonstrated to be strongly catalyzed by the presence of heparin and pre-aggregated synthetic Tau-fragments. Monomeric Tau-fragments (TauMono) were incubated at $37{ }^{\circ} \mathrm{C}$ under shaking conditions, and ThioT fluorescence was measured $1 \mathrm{~h}, 24 \mathrm{~h}$ and 3 days post-incubation with, respectively, heparin, pre-aggregated Tau-seeds, pre-aggregated $\mathrm{A} \beta$-seeds, and the respective buffers of the seeds as control. Quantitative analysis at the different time points post-seeding revealed that $A \beta$-seeds induced significant Tau-aggregation at 3 days post-seeding (Fig. 3). Further controls were performed with albumin (a non-amyloidogenic protein) and with pre-aggregated amylin (a well-characterized unrelated amyloid protein) [9], revealing no significant induction of Tau-aggregation 3 days post-seeding (Fig. S3). Tau-aggregation induced by pre-aggregated $A \beta$-seeds was slower compared to induction by pre-aggregated Tau-seeds, but markedly and significantly increased compared to the buffer conditions (Fig. 3). This finding was further consistently reproduced in all subsequent experiments performed for follow-up experiments, since it was used as a primary control analysis of the samples. Next, direct $\mathrm{A} \beta$-seeded Tau-aggregation was further confirmed using a sedimentation assay. Following incubation of the different conditions for 3 days, high-speed centrifugation was performed to pellet aggregated forms of Tau. Subsequently, pelleted and soluble forms of Tau were analyzed by Western blotting (Fig. 3). This analysis further confirmed the results obtained with ThioT, demonstrating 
Fig. 3 Pre-aggregated $A \beta$-seeds heterotypically seed aggregation of monomeric Tau in a cell-free assay, demonstrated by ThioT and sedimentation analysis. a Schematic presentation of the different experimental conditions of incubation. Synthetic pre-aggregated $\mathrm{A} \beta$ peptides, synthetic pre-aggregated Tauseeds and their respective buffer conditions are co-incubated with monomeric Tau-fragments $\mathrm{Tau}^{\mathrm{Mono}}$. b Aggregation of TauMono by Tau-seeds and $A \beta$-seeds was measured using ThioT assay at $1 \mathrm{~h}, 1$ day and 3 days post co-incubation. Quantitative analysis revealed significant induction of Tau-aggregation by $\mathrm{A} \beta$-seeds and Tau-seeds $(n=9$; two-way ANOVA followed by Bonferroni post hoc test; three independent experiments; mean \pm SEM are presented; $* * p$ value $<0.01, * * * p$ value $<0.001)$. $\mathbf{c}$ The presence of Tau-aggregates following co-incubation of Tau ${ }^{\text {Mono }}$ with either Tau-seeds or $\mathrm{A} \beta$-seeds was demonstrated using a Tauaggregates sedimentation assay, followed by Western blotting. Supernatant (S) and pellet (P) obtained following $100,000 \mathrm{~g}$ centrifugation of co-incubated samples (3 days co-incubation) were analyzed by immunoblotting with Dako-Tau antibody

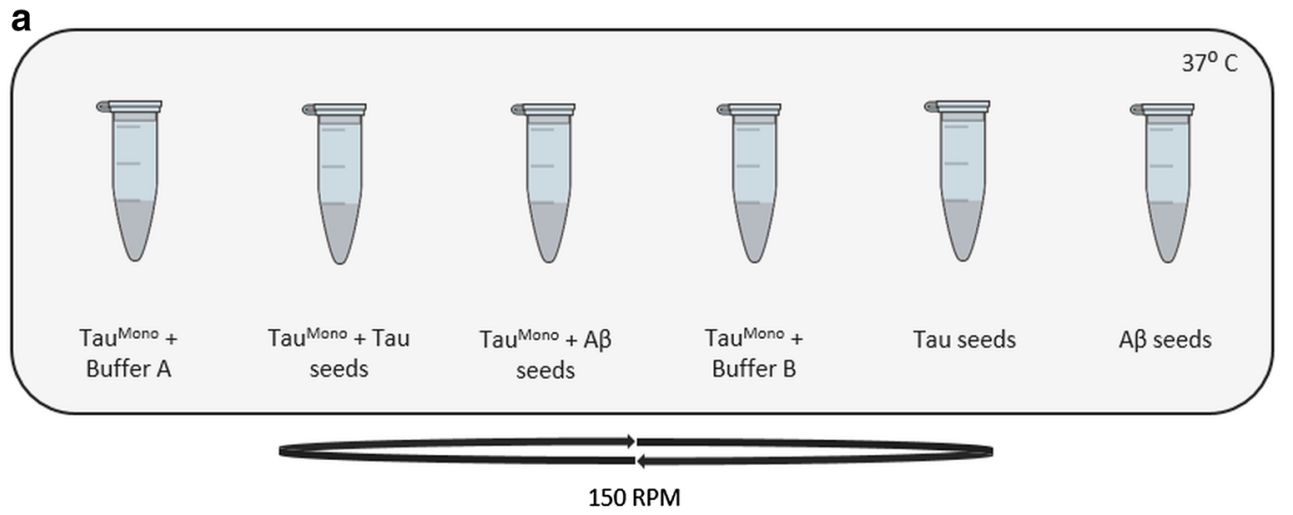

b

ThT Assay

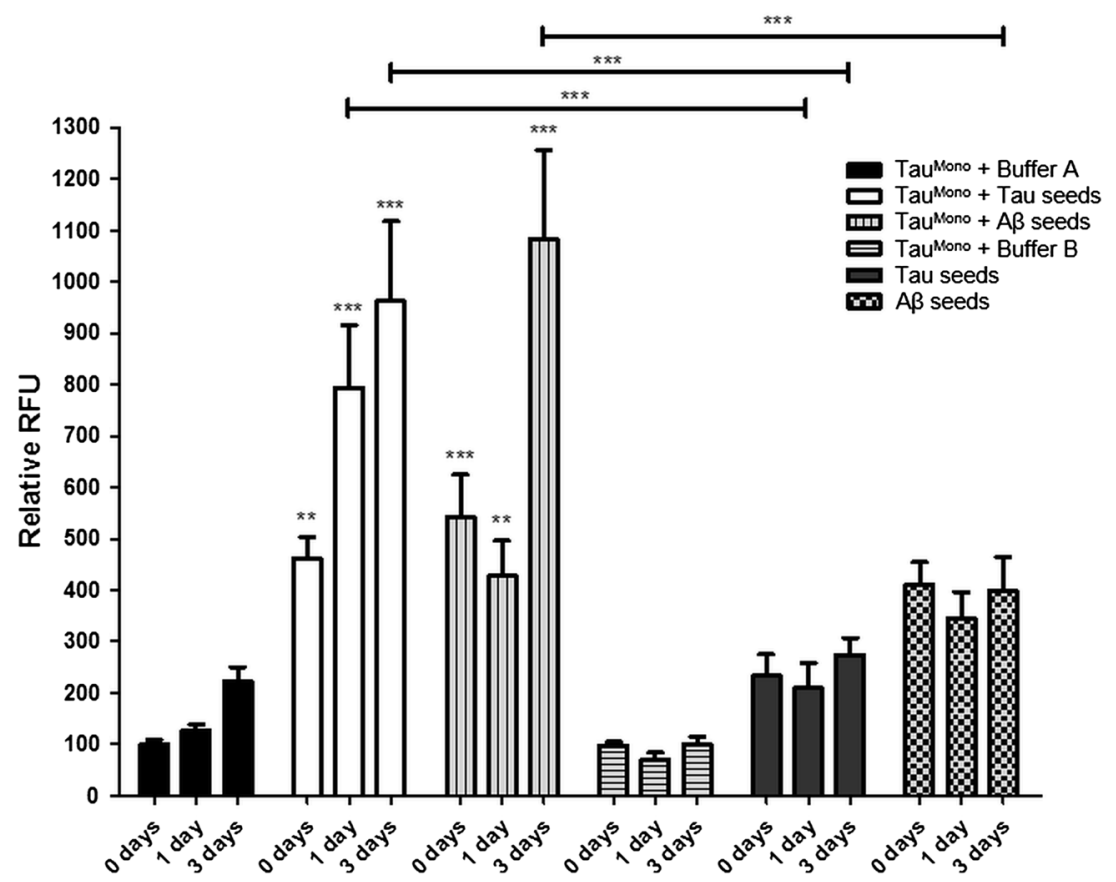

C

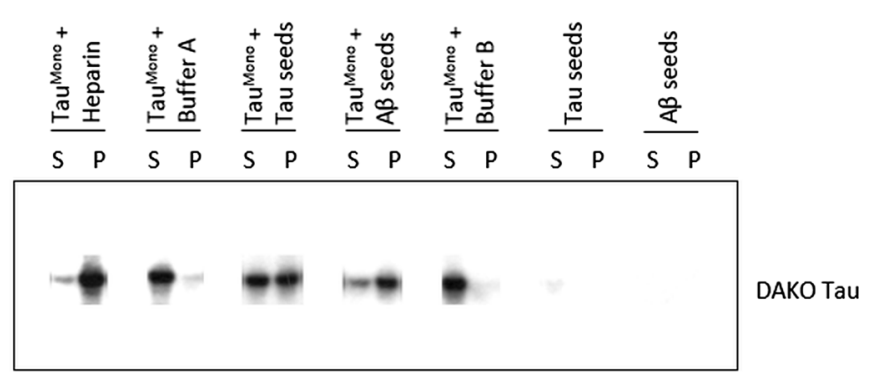

that $\mathrm{A} \beta$-seeds induced strong Tau-aggregation 3 days post-co-incubation.

To analyze the structure of $\mathrm{A} \beta$-seeded Tau-aggregates, we performed negative-staining electron microscopic analysis (EM) in combination with immuno-EM using a Tauspecific antibody 3 days post-incubation. This revealed the abundant presence of 10- to 15-nm wide fibrillar Tau aggregates in samples incubated with heparin, pre-aggregated Tau-seeds and pre-aggregated $\mathrm{A} \beta$-seeds, while filaments were nearly absent in buffer only conditions (Fig. 4). Strong induction of fibrillar Tau-aggregates was demonstrated following induction of Tau-aggregation with heparin. Fibrillar 
Tau-aggregates were also abundantly detected following seeding with pre-aggregated synthetic Tau-seeds, yet less strong as with heparin (Fig. 4). This is reflected in the fact that non-fibrillar and smaller globular Tau-forms were also still detected. Importantly, incubation with $A \beta$-seeds induced abundant formation of Tau-fibrils to comparable extents as following addition of Tau-seeds (Fig. 4). These fibrils were identified by staining with immuno-EM as Taufibrils, using a Tau-specific antibody. The specificity of this staining was demonstrated by appropriate control stainings, with buffer only (no Tau) and without primary antibody revealing no staining (Fig. S4). Furthermore, increasing dilutions of Tau, demonstrated decreasing staining with lower concentrations, further highlighting the specificity of the staining (Fig. S4). These data clearly demonstrate that pre-aggregated $A \beta$ can seed filamentous Tau-aggregation following direct co-incubation. These data thereby prove heterotypic seeding of filamentous Tau aggregation by $\mathrm{A} \beta$.

We next performed double immuno-EM analysis using anti-A $\beta$ and anti-Tau antibodies, to demonstrate cross-seeding. This clearly demonstrated the combined presence of $\mathrm{A} \beta$ and Tau in the $\mathrm{A} \beta$-induced Tau-filaments, demonstrating cross-seeding of Tau by pre-aggregated $\mathrm{A} \beta$ (Fig. 5). Specificity of the $A \beta$ staining was demonstrated by strong specific staining of pre-aggregated $A \beta$ and appropriate negative controls (buffer only and no primary antibody, revealing absence of staining) (Fig. S4). Using this staining, anti-A $\beta$ stained structures were detected at limited spots within or at the basis of the Tau-stained filaments (Fig. 5). This is in line with $\mathrm{A} \beta$-seeds providing a nucleus for subsequent Tau-fibril formation. Following $A \beta$-induced seeding the Tau-filaments seem to be 'preferentially' elongated by Tau rather than $A \beta$.

\section{Heterotypic A $\beta$-seeded Tau provides potent seeds for induction and propagation of Tau-pathology in vitro and in vivo}

Having demonstrated that pre-aggregated $\mathrm{A} \beta$ can heterotypically seed Tau filament formation, we analyzed the potential of these $A \beta$-induced Tau-aggregates to seed Tau-pathology in vitro. $A \beta$-seeded Tau-aggregates were used to seed Tau-aggregation in the above described Tauaggregation assay in QBI-293 cells (Fig. 6). This revealed the potential of $\mathrm{A} \beta$-induced Tau-seeds (further denoted as $\mathrm{A} \beta$-seeded Tau) to seed Tau-pathology in the cellular Tauaggregation assay. Tau-aggregates induced by $\mathrm{A} \beta$-seeded Tau, strongly stained with AT8 and MC1 antibodies, revealing the presence of hyperphosphorylated, conformationally altered Tau aggregates. Comparative quantitative analysis revealed a significantly higher induction of Tau-aggregation compared to homotypic Tau-seeds, further highlighting that these seeds efficiently induce Tau-aggregation in vitro (Fig. S5).
Fig. 4 Pre-aggregated $A \beta$-seeds heterotypically seed fibrillization of monomeric Tau in a cell-free assay, demonstrated by immuno-electron microscopy analysis. The different conditions of co-incubation analyzed with the ThioT assay and sedimentation assay were subsequently analyzed by immuno-electron microscopy with anti-Tau antibody (DAKO-Tau; $10 \mathrm{~nm}$-gold labeled). This demonstrated strong induction of Tau fibrillization following direct co-incubation of Tauseeds with Tau ${ }^{\text {Mono }}$ as demonstrated previously. Co-incubation of $\mathrm{A} \beta$-seeds with $\mathrm{Tau}^{\mathrm{Mono}}$ very strongly resulted in Tau-fibrillization. Incubation of Tau ${ }^{\text {Mono }}$ with the respective buffers did not induce Taufibrils; only globular structures were detected. Higher magnifications of $A \beta$-seed-induced Tau fibrils and Tau-seed-induced Tau fibrils are presented (scale bar $100 \mathrm{~nm}$ )

We next analyzed the seeding potential of $\mathrm{A} \beta$-seeded Tau in vivo by injection in TauP301S transgenic mice [58], using a similar approach as previously published by us and others $[22,51]$. Due to the procedure of heterotypic seeding used in this work, five times more diluted final concentrations of Tau-seeds needed to be used compared to our previous work [51]. Induction of Tau-aggregation was analyzed 3 months post-injection following intracerebral injections in frontal cortex and hippocampus, using optimized protocols in our group [39, 51]. Our analysis indicated a strong induction of Tau-pathology by heterotypic $\mathrm{A} \beta$-seeded Tau compared to non-seeded monomeric Tau or buffer injections only, 3 months post-injection (Fig. S6). We furthermore compared these newly identified heterotypic A $\beta$-seeded Tau seeds with homotypic Tau-seeds, which have been shown to be potent inducers of Tau-aggregation and propagation of Tau-pathology in transgenic mice in vivo [51]. Quantitative analysis revealed that $A \beta$-seeded Tau even more potently induced Tau aggregation than homotypic Tau-seeds (Fig. 7), thereby highlighting the high potency and efficiency of these seeds to induce Tau-aggregation in vivo. Tau-aggregates induced by $A \beta$-seeded Tau seeds were stained with antibodies recognizing pathological forms of Tau, i.e. AT8, MC1 and AT100. Tau-aggregates were furthermore stained following Gallyas silver staining and ThioS staining, revealing the true fibrillary nature of these aggregates. A $\beta$-staining did not reveal any signal, demonstrating that these aggregates are mainly composed of Tau.

To further assess if $\mathrm{A} \beta$-seeded Tau-seeds induced spreading or propagation of Tau-pathology, we performed an immunohistochemical analysis of Tau-pathology of the contralateral side, following unilateral seeding. We have previously demonstrated spreading of Tau-pathology following injections in different brain regions, i.e. entorhinal cortex, basal ganglia and combined hippocampal/cortical injections, to functionally connected brain regions [51]. We here demonstrate that unilateral injection of $A \beta$-seeded Tau-seeds resulted in propagation of Tau-pathology to the contralateral side. Tau-pathology was significantly induced 

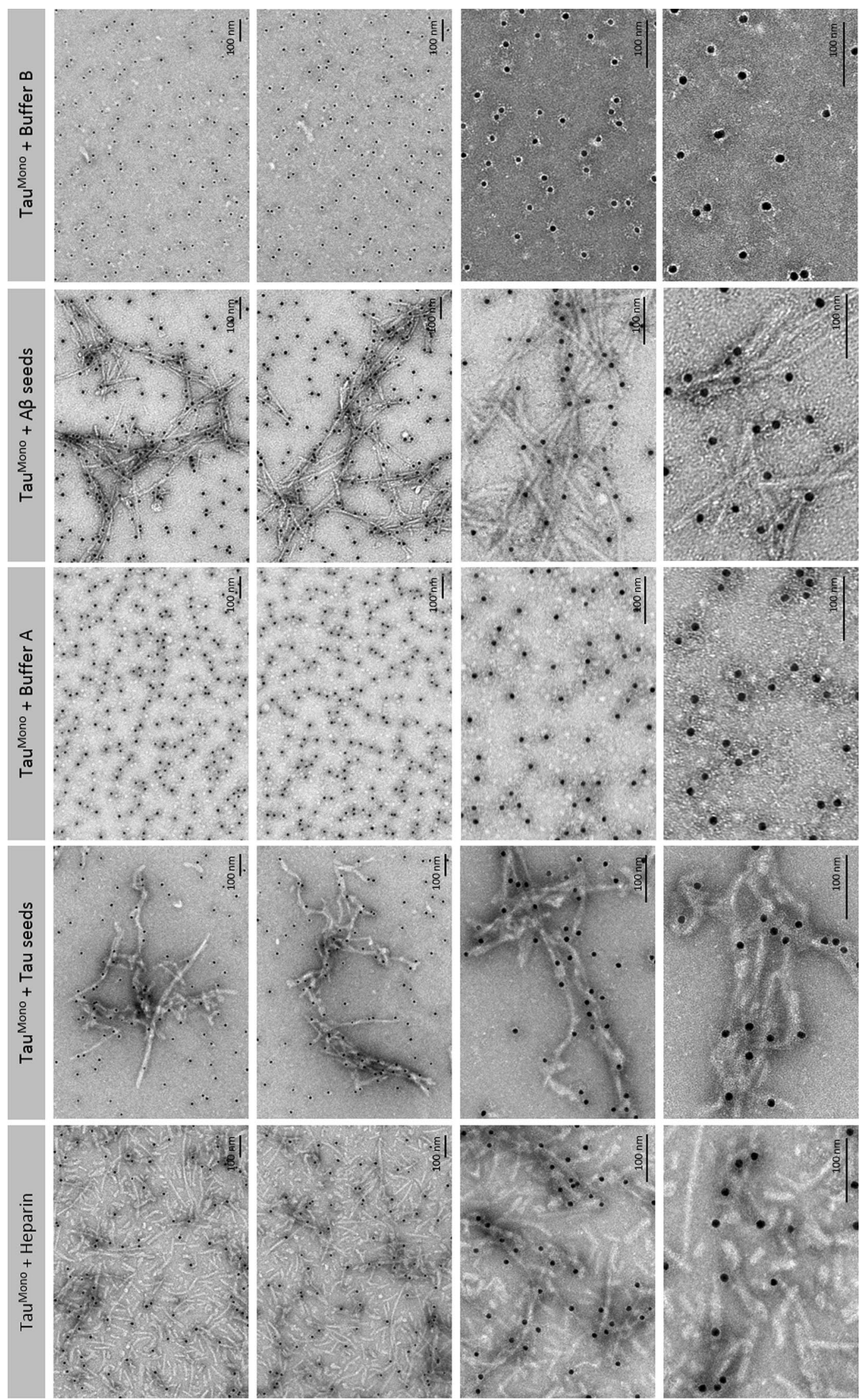


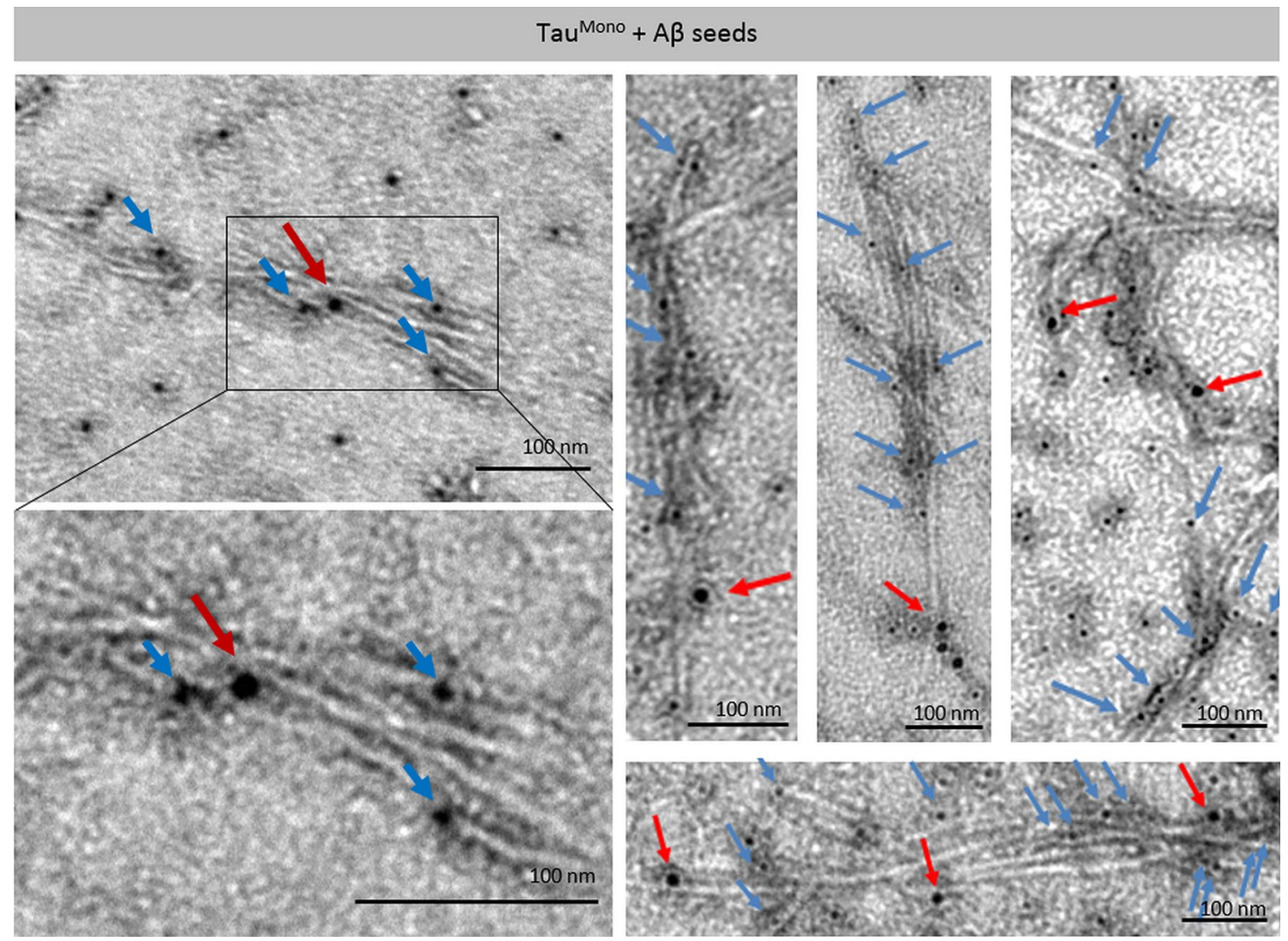

Fig. 5 Pre-aggregated $A \beta$ provides seeds for Tau-fibrillization in a cell-free assay, demonstrated by immuno-electron microscopy analysis. Double-immuno-EM analysis using anti-A $\beta$ antibody (WO2; $10 \mathrm{~nm}$-gold labeled; red arrows) and anti-Tau antibody (DAKOTau; $5 \mathrm{~nm}$-gold labeled, blue arrows), reveals the presence of $\mathrm{A} \beta$

compared to vehicle injected mice, but remained less prominent compared to the ipsi-lateral side, as demonstrated by a semi-quantitative heat-map analysis (Fig. 8) and by quantitative analysis (Fig. S7), in line with propagation from the injected side. Together, our data indicate that $A \beta$-seeded Tau provides strong seeds for induction and propagation of Tau-pathology.

Together, our data demonstrate heterotypic seeding of filamentous Tau-aggregation by pre-aggregated $A \beta$. $\mathrm{A} \beta$-seeds thereby provide seeds for Tau-aggregation, strongly accelerating the lag-phase for Tau-aggregation. These newly identified heterotypic $\mathrm{A} \beta$-seeded Tau-seeds provide potent seeds for induction and propagation of Taupathology in vivo.

\section{Discussion}

In this work we have demonstrated (1) direct seeding of Tau-fibrillization by pre-aggregated $\mathrm{A} \beta$ in a cell-free assay, demonstrating filamentous Tau-aggregation by heterotypic within Tau-filaments. $A \beta$ is scarcely detected per fibril compared to the abundant detection of Tau, suggesting that $A \beta$-seeds provide a nucleus, which is subsequently preferably extended by Tau ${ }^{\text {Mono }}$ (scale bar $100 \mathrm{~nm}$ )

Fig. $6 \mathrm{~A} \beta$-induced Tau-aggregates provide potent seeds for prionlike Tau-aggregation in cells in vitro. a Schematic presentation of the assay. Induction of Tau-aggregation was performed using heterotypic $\mathrm{A} \beta$-seeded Tau ${ }^{\mathrm{Mono}}$ as "seeds" in TauP301L-GFP expressing cells, to analyze their propagating potential. Analysis is performed by cytological analysis following stringent detergent extraction to eliminate soluble forms of Tau. b This analysis demonstrated efficient induction of Tau-aggregation following seeding with $\mathrm{A} \beta$-seeded Tau ${ }^{\mathrm{Mono}}$, reflected in strong induction of aggregated Tau, phosphorylated at pathologically relevant epitopes (AT8) and conformationally altered (MC1). Representative images of GFP-signal following stringent extraction with $1 \%$ TX-100 and immunofluorescent staining using antibodies recognizing phosphorylated Tau (S202/T205) (AT8) and conformationally altered Tau (MC1) are presented, in non-seeded conditions and following seeding with $\mathrm{A} \beta$-seeded aggregated Tau (scale bar $100 \mu \mathrm{m})$

seeding with $\mathrm{A} \beta$-seeds. We furthermore demonstrate (2) induction of Tau-aggregation and acceleration of incipient Tau-aggregation by $\mathrm{A} \beta$-seeds in a well-characterized cellular assay of Tau-aggregation and (3) finally we analyzed the repercussions of these heterotypic $A \beta$-seeded Tau in vitro and in vivo. We thereby demonstrate that heterotypic 
a In vitro induction of Tau-aggregation

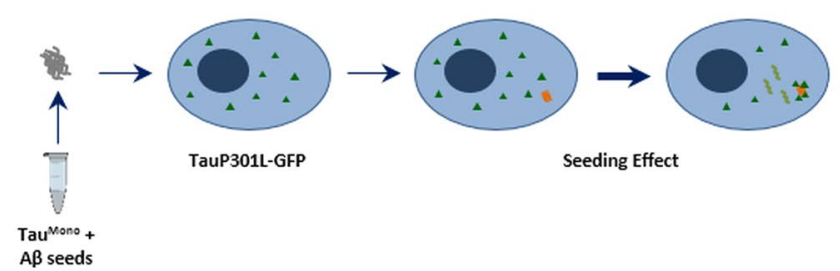

b
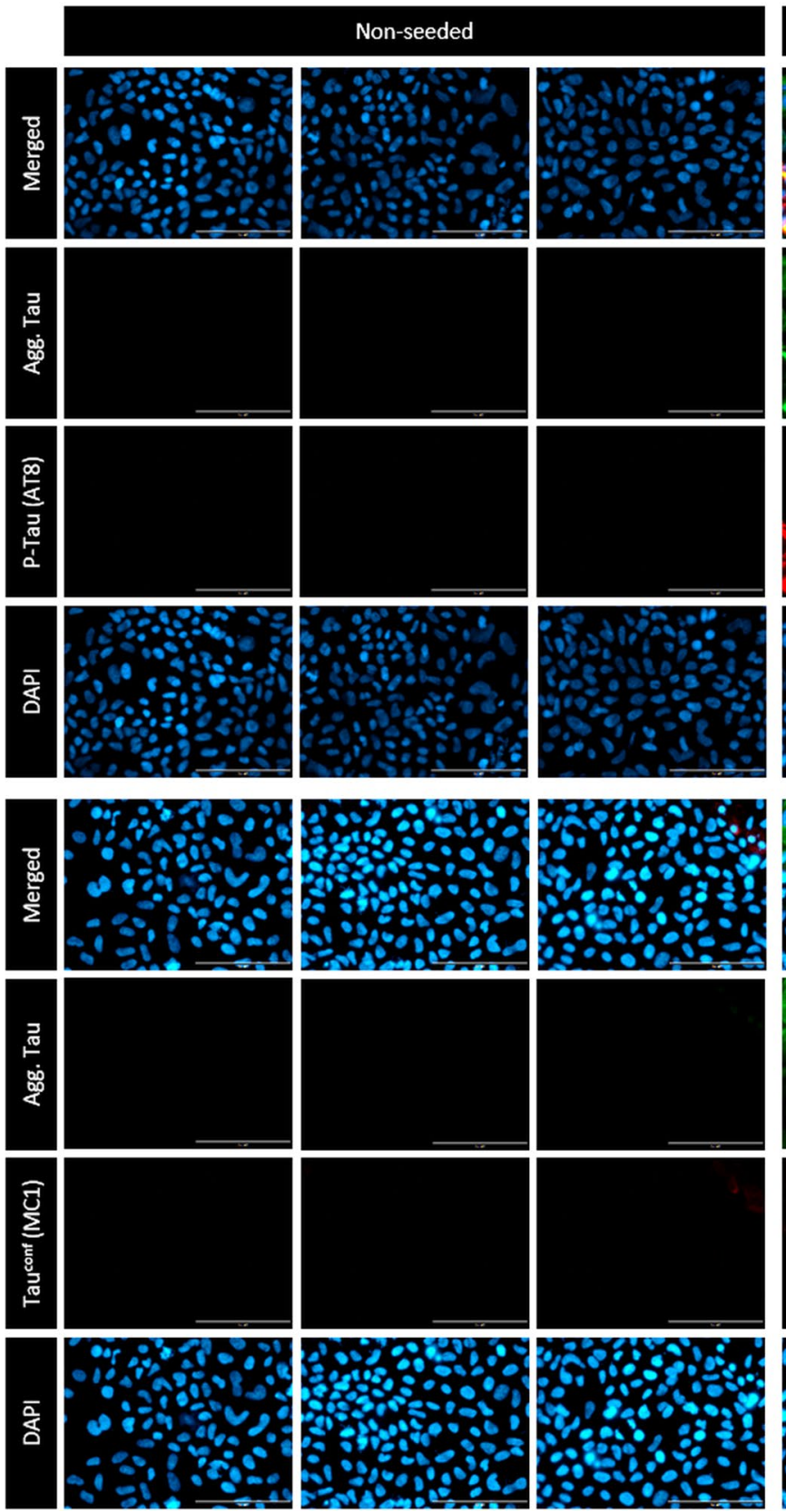
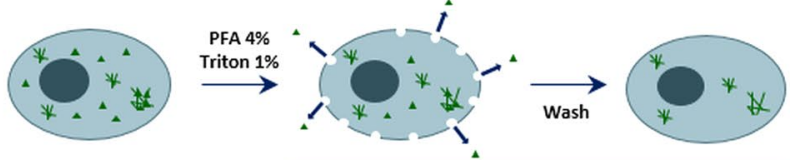

Green signal = Tau aggregation
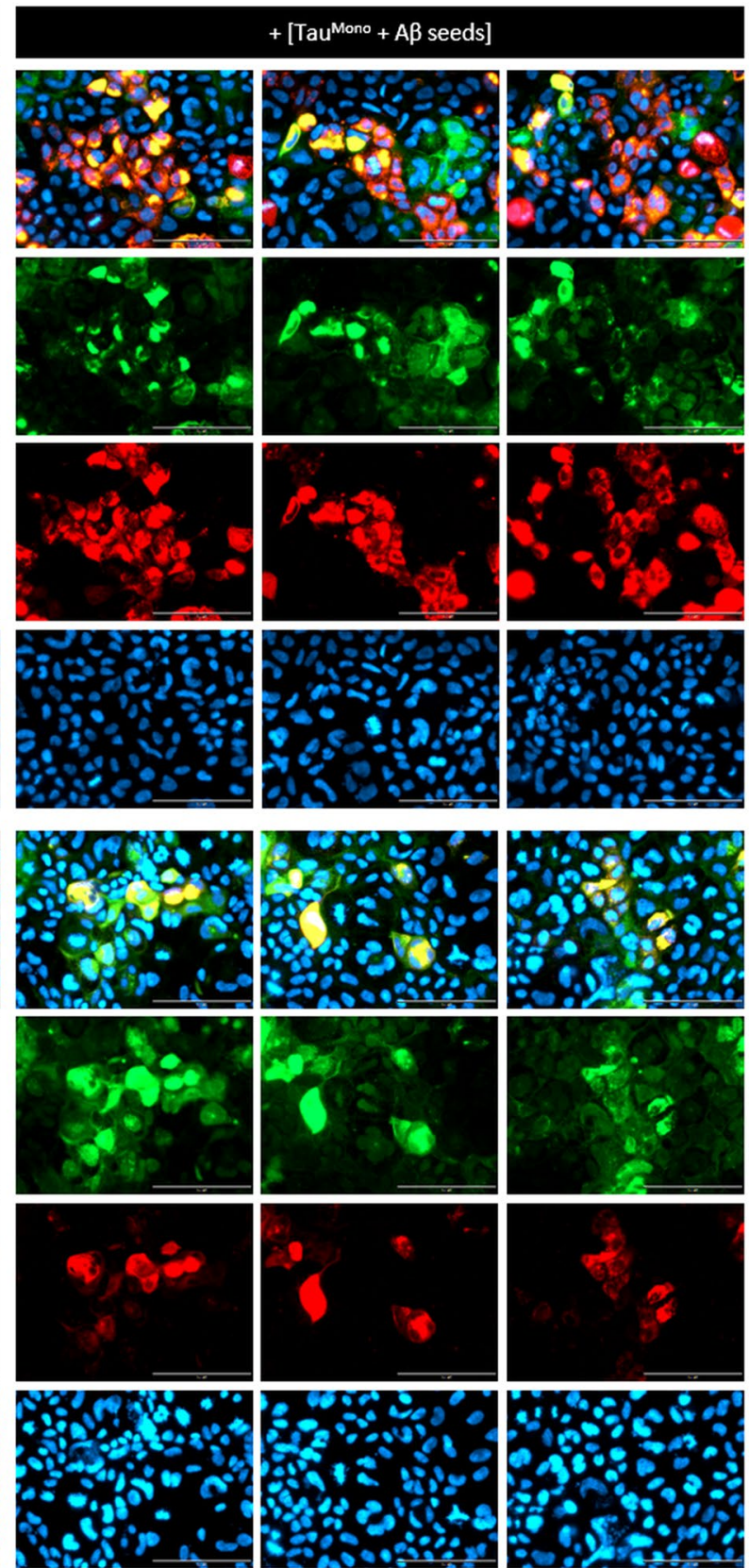
a In vivo induction of Tau-aggregation

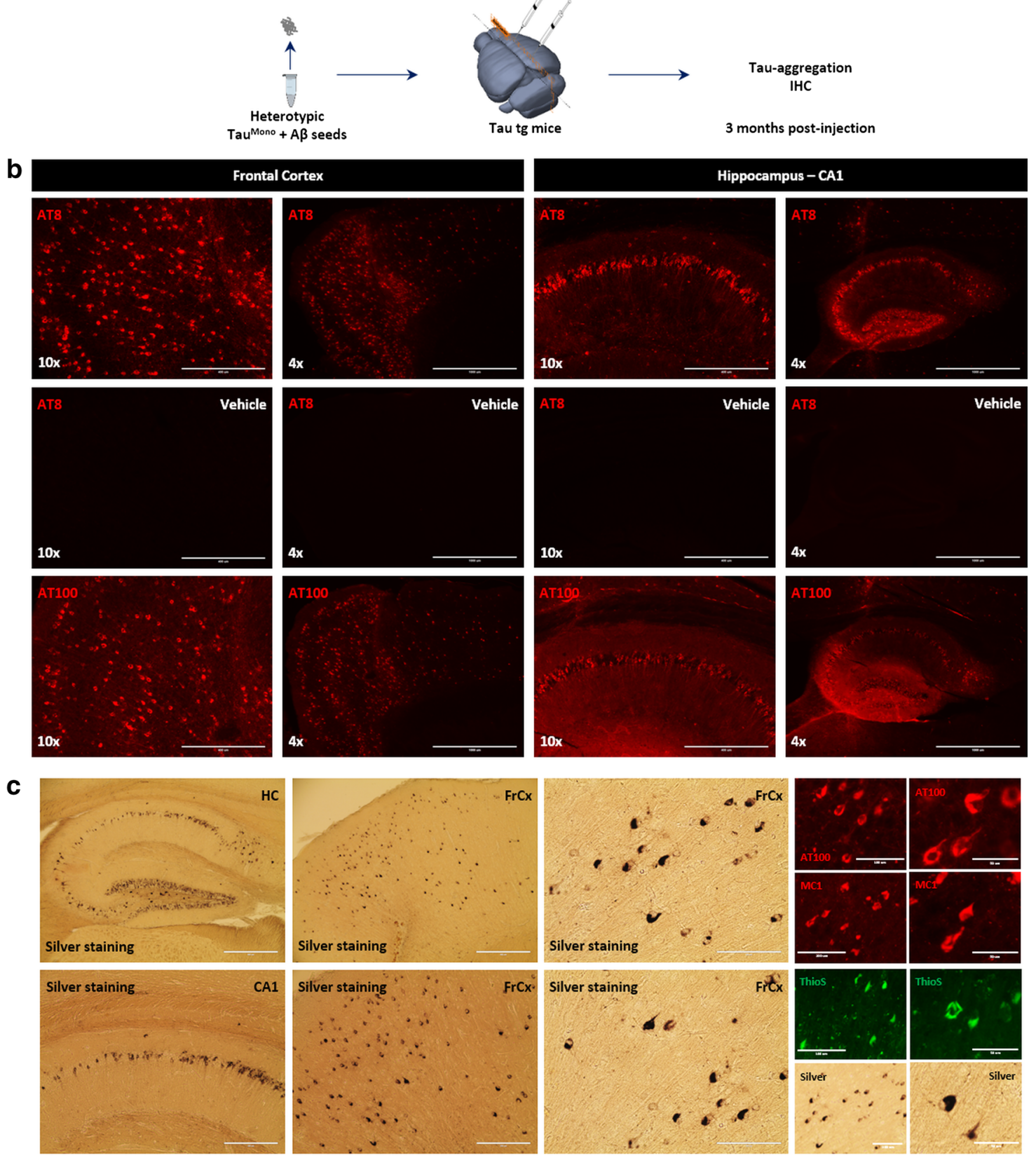

d

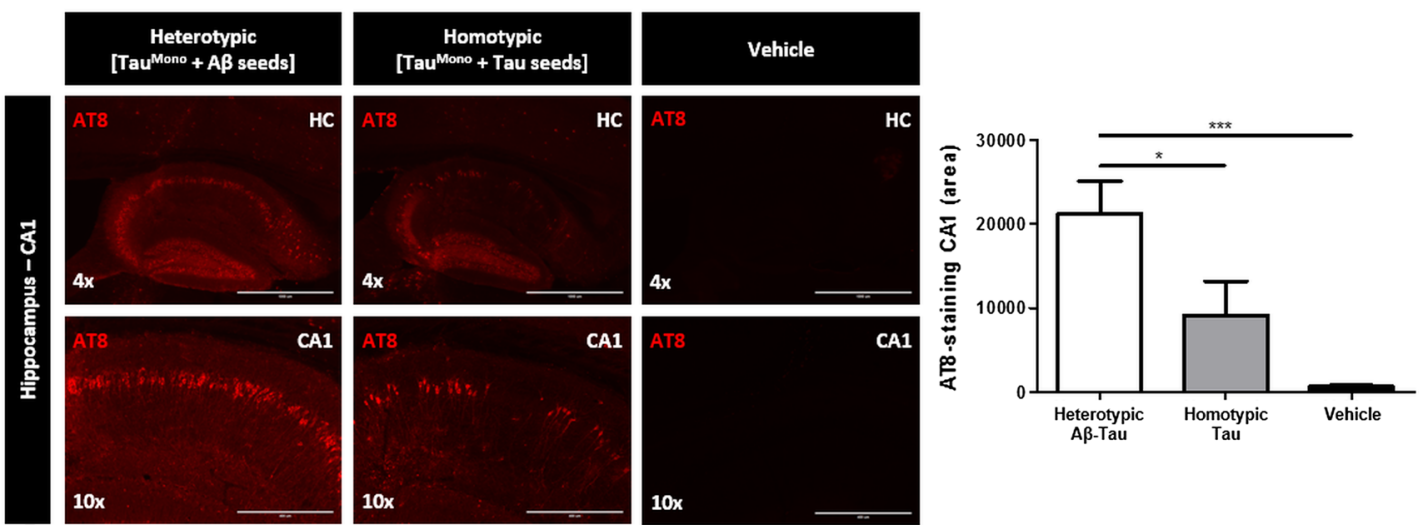


४Fig. 7 Heterotypic $A \beta$-seeded Tau efficiently induces Tau-aggregation in Tau transgenic mice in vivo. a Schematic presentation of the assay: Induction of Tau-aggregation using heterotypic $A \beta$-seeded $\mathrm{Tau}^{\mathrm{Mono}}$ as "seeds" in Tau transgenic mice. Tau transgenic mice (4 months old) were intracerebrally injected in frontal cortex and hippocampus and analyzed 3 months post-injection using AT8 to assess induction of Tau-pathology in frontal cortex and hippocampus. b Representative images of AT8 and AT100 staining in frontal cortex and hippocampus are shown following injection with heterotypic $\mathrm{A} \beta$-seeded Tau ${ }^{\text {Mono }}$ (further denoted heterotypic $\mathrm{A} \beta$-seeded Tau) $(4 \times$ and $10 \times$ magnifications are shown, scale bar 1000 and 400 $\mu \mathrm{m}$, respectively). No AT8 staining was detected in vehicle injected mice, following concomitant staining and imaging. $\mathbf{c}$ Tau-pathology induced by $\mathrm{A} \beta$-seeded Tau is strongly stained with AT8, AT100, $\mathrm{MC} 1$, Gallyas silver staining and ThioS staining. This demonstrates the true fibrillar nature of NFTs induced by $\mathrm{A} \beta$-seeded Tau injections. Concomitant stainings with AT8, AT100, MC1 and silver staining in vehicle injected mice revealed no staining in Tau transgenic mice, 3 months post-injection (data not shown). d Comparative analysis of seeding efficiency of heterotypic A $\beta$-seeded Tau and homotypic Tauseeds in Tau transgenic mice at 3 months post-injection. Quantitation of AT8 staining demonstrated increased AT8 staining following injections with heterotypic $\mathrm{A} \beta$-seeded Tau compared to homotypic Tauseeds, while Tau-pathology was significantly induced in both conditions compared to vehicle injected mice $(n=6$ mice per condition; one-way ANOVA followed by Tukey's post hoc test; mean \pm SEM are presented; * $p$ value $<0.05, * * * p$ value $<0.001$ )

seeded Tau by $\mathrm{A} \beta$ provides potent seeds to induce and propagate Tau-aggregation in vivo. Taken together, we demonstrate that pre-aggregated $A \beta$ can template filamentous Tau aggregation by cross-seeding, providing potent seeds for induction and propagation of Tau-pathology in a prion-like way in vivo. This presents a compelling molecular mechanism of $\mathrm{A} \beta$-induced propagation of Tau-pathology in AD.

In this work, we demonstrate direct seeding of Taufibrillization by pre-aggregated $A \beta$ in a cell-free assay. Prion-like seeding mechanisms have gained important interest as pathogenetic mechanism in a variety of neurodegenerative proteinopathies $[6,8,12,16,17,22,27,28,30$, $33,38,39,51,56]$. Homotypic seeding and prion-like properties have been demonstrated for different proteins associated with these neurodegenerative disorders $[6,8,12,16$, 17, 22, 27, 28, 30, 33, 38, 39, 51, 56] including Tau. Heterotypic seeding or cross-seeding has been demonstrated for Tau and alpha-synuclein, and for different combinations of amyloidogenic proteins in vitro [11, 14, 15, 35]. Direct cross-seeding between pre-aggregated $\mathrm{A} \beta$ and Tau is supported by demonstrating direct binding between $A \beta$ peptides and Tau [18] and in silico models for $A \beta$-induced beta-sheet formation of Tau-fragments [34]. However, we here demonstrate direct induction of Tau fibrillization by pre-aggregated $\mathrm{A} \beta$-seeds, in an unequivocal way using ThioT assay, sedimentation analysis and immuno-EM. Having demonstrated the induction of Tau-fibrillization in a cell-free assay, we further extended this analysis in a wellcharacterized cellular Tau aggregation assay. In this assay, the nucleation-dependent initial lag phase for Tau-fibrillization in cells is proposed to be by-passed by the addition of preformed pre-aggregated synthetic Tau-seeds, which accelerate fibrillization of monomeric Tau. This demonstrated that pre-aggregated $A \beta$ was able to seed Tau-aggregation, similarly as Tau-seeds, albeit less efficient. Previous data demonstrated the occurrence of homotypic seeding for $\mathrm{A} \beta$, alpha-synuclein, Tau and huntingtin in a cellular Tau aggregation assay, while heterotypic cross-seeding was not observed [44]. The observed difference in our current dataset may rely on the fact that homotypic seeding is much more efficient compared to heterotypic seeding, thereby masking the effect of heterotypic seeding in the previous study which was focused on homotypic seeding, or may rely on the properties of the $A \beta$-seeds used or assay sensitivity. In favor of this is the demonstration of heterotypic seeding of alpha-synuclein and Tau, in a similar Tauaggregation assay and in primary neurons [15, 57], further corroborating the potential of heterotypic seeding between prion-like proteins in cellular assays. Clearly, in our assay heterotypic seeding was much less efficient compared to homotypic seeding. Interestingly, $A \beta$-seeds potently induced Tau-aggregation in the cellular Tau-aggregation assay, when incipient Tau-aggregates were present. This finding is reminiscent and intriguing in the context of $\mathrm{AD}$, as outlined below. Furthermore, inflammatory processes or neuron-specific mechanisms can be mechanistically excluded for $\mathrm{A} \beta$-induced Tau-aggregation in this assay, since a non-neuronal cell line is used. Our assay suggests that pre-aggregated $A \beta$-seeds provide seeds for templating Tau-aggregation, bypassing the required nucleation phase for Tau-aggregation. Neither monomeric $\mathrm{A} \beta$ nor monomeric Tau forms induced Tau-aggregation in this assay, further strengthening templated seeding as the mechanism involved. Finally, we demonstrated that heterotypically aggregated Tau by $\mathrm{A} \beta$, provided potent and efficient seeds for induction and propagation of Tau-aggregation in vivo in Tau transgenic mice. Tau-pathology induced by $A \beta$-seeded Tau was characterized by immunostaining with antibodies recognizing Tau phosphorylated at pathologically relevant epitopes, i.e. AT8, AT100 and recognizing conformationally altered Tau. Tau aggregates were stained by Gallyas silver staining and ThioS staining, demonstrating the true fibrillary nature of these aggregates. Tau pathology propagated efficiently from the injected brain hemisphere to the contralateral side, in a similar pattern as previously observed [39, 51]. Our data thereby demonstrate the strong potential of heterotypic $A \beta$-seeded Tau to seed and propagate Tau-pathology in vivo, strengthening the relevance of these newly identified seeds.

Our data indicate a compelling mechanism for $\mathrm{A} \beta$-induced Tau-pathology in preclinical models of $\mathrm{AD}$. Since $A \beta$-induced Tau-pathology is situated at the effective 
a

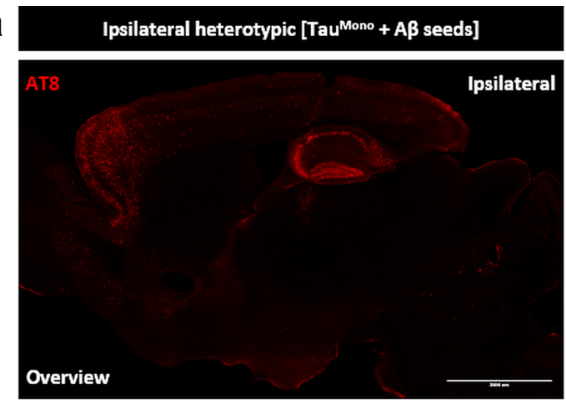

b
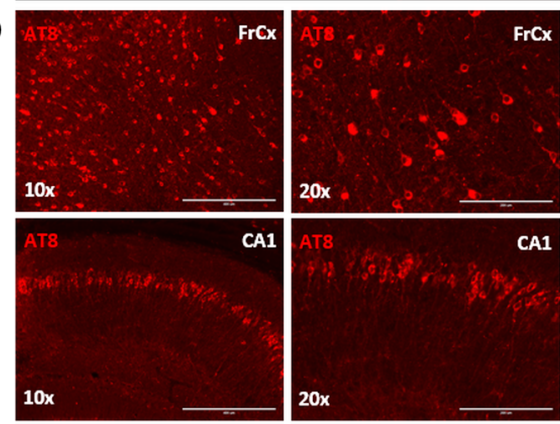

C

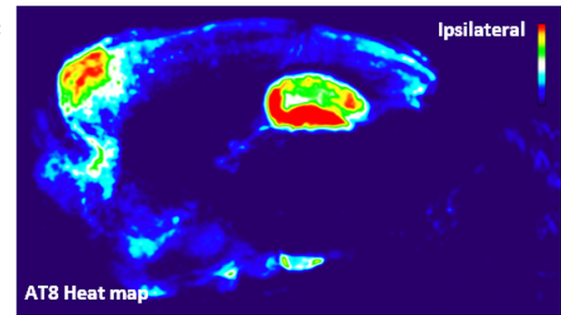

Contralateral heterotypic [Taumono $+A \beta$ seeds]

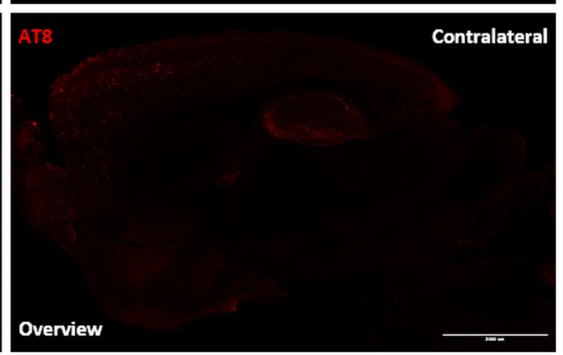

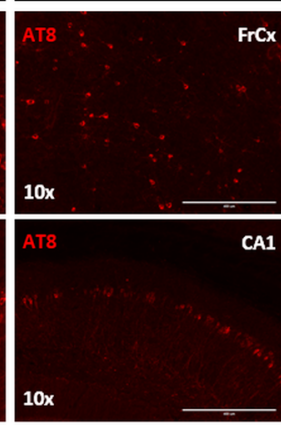

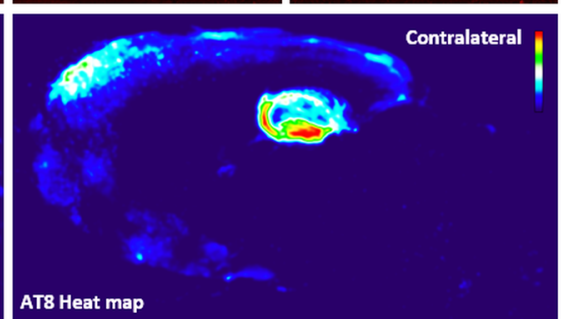

20x

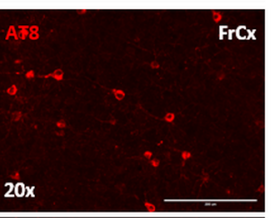

CA1
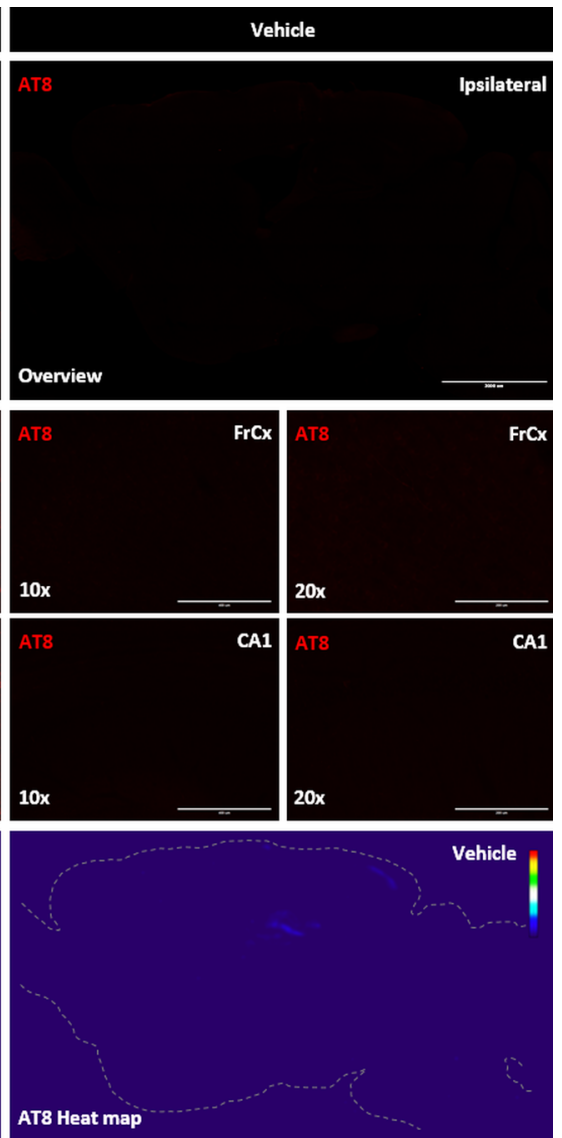

Fig. 8 A $\beta$-seeded Tau propagates Tau pathology along functional connections in Tau transgenic mice in vivo. a Representative AT8 stainings of sagittal brain sections of heterotypic $\mathrm{A} \beta$-seeded Tau in frontal cortex and hippocampus $(\mathrm{FrCx} / \mathrm{HC})$, from the ipsi-lateral (injected) hemisphere and the contralateral (non-injected) hemisphere in comparison with vehicle injected brains of Tau transgenic mice 3 months post-injection, are shown (scale bar $2000 \mu \mathrm{m})$. b Higher magnifications of frontal cortex and hippocampal regions of the ipsi-lateral side and contralateral side, of mice injected with heterotypic $\mathrm{A} \beta$-seeded Tau in frontal cortex and hippocampus and vehicle injected Tau mice are presented $(10 \times, 20 \times$ magnifications are shown; scale bar 400 and $200 \mu \mathrm{m}$, respectively). This reveals clear-cut induction of Tau-pathology, at the contralateral side of heterotypic $A \beta$-seeded Tau injected mice, compared to vehicle injected brains, but less strong compared to the injected side, demonstrating propagation of Tau-pathology to remote but functionally connected brain regions. Tau-pathology stained with AT8 correlates strongly

beginning of $\mathrm{AD}$, i.e. the conversion from preclinical stages to $\mathrm{MCI}$ and subsequently to $\mathrm{AD}$, it is considered as a keypathogenetic mechanism. Its understanding is crucial to understand $\mathrm{AD}$ pathogenesis and design of therapeutic strategies $[3,19,25,26,46,47,53,54]$. A $\beta$-induced Tauaggregation has been reproducibly and robustly recapitulated in a variety of different in vivo preclinical models using different experimental approaches [2, 13, 20, 21, 29, 36, 40, 45, 49, 50]. Thus, A $\beta$-induced Tau-pathology was demonstrated by (1) crossing or co-expressing of mutant with Gallyas silver staining and ThioS (results not shown). c To analyze propagation of Tau pathology semi-quantitatively, averaged heat maps representing color-scored, averaged images of AT8 stained Taupathology following injection of heterotypic $A \beta$-seeded Tau in frontal cortex and hippocampus ( $\mathrm{FrCx} / \mathrm{HC})$ and vehicle injections were generated. Averaged heat maps of AT8 stained Tau-pathology at the side of injection (denoted "ipsi-lateral") and the contralateral non-injected side (denoted "contralateral") were generated. Heat maps were generated by averaging AT8 stainings of multiple mice for each injection paradigm using Image $\mathrm{J}$ ( $n=6$ for each condition). This reveals clear-cut induction of averaged Tau-pathology in the non-injected hemisphere of heterotypic $A \beta$-seeded Tau-injected brains (in frontal cortex and hippocampus), absent in age-matched vehicle injected mice, but less strong compared to the injected brain hemisphere, demonstrating propagation of Tau-pathology to remote but functionally connected brain regions

APP/PS1 with mutant Tau in transgenic mice [2, 29, 36, 40, 49] following (2) injection of pre-aggregated $A \beta$ containing extracts derived from AD patients or APP/PS1 transgenic mice [2], or (3) following injection of synthetic pre-aggregated $\mathrm{A} \beta$ [13]. Different cellular and molecular mechanisms must be considered for $\mathrm{A} \beta$-induced Tau-pathology in these models, including neuron-specific and synaptic/ synaptotoxic mechanisms [13], or a role of $\mathrm{A} \beta$-induced inflammation and other potential mechanisms $[13,23$, $24,48,49]$. Our current data demonstrating that $A \beta$-seeds 
can heterotypically induce filamentous Tau-aggregation, providing potent seeds for induction of Tau-aggregation, demonstrate an attractive mechanism for $\mathrm{A} \beta$-induced Tau-aggregation in these preclinical in vivo models. Interestingly, in these preclinical models pre-aggregated $A \beta$ strongly induced Tau-pathology at the site of injection but also in remote brain regions functionally connected to the injection site [2, 13, 40, 50] (Fig. 9). The induction and spreading of Tau-pathology in these preclinical models in functionally connected brain regions is reminiscent of prion-like Tau-seeding [1, 7, 22, 27, 51]. Tau-pathology is thereby propagated to remote but functionally connected brain regions, affecting intrinsic functional neuronal networks, reminiscent of the networks affected in $\mathrm{AD}$ and related Tauopathies $[1,22,51]$. We here propose heterotypic seeding of Tau by $A \beta$ as a contributing mechanism of $A \beta$-induced Tau-pathology in these preclinical models. It must be noted that prion-like seeding is mechanistically not yet fully resolved, in terms of uptake and spreading of "seeds", nor for Tau-seeds, nor in the case of cross-seeding for $\mathrm{A} \beta$-seeds. Nevertheless, prion-like seeding has been reproducibly recapitulated in different models using different types of "seeds". Taken together, the heterotypic seeding of filamentous Tau aggregation, along functional connections, providing potent seeds for Tau-aggregation as demonstrated in this work, presents as an attractive molecular mechanism of $A \beta$-induced propagation of Tau pathology, in functionally connected brain regions as observed in preclinical AD models (Fig. 9).

Heterotypic seeding of Tau-pathology by $A \beta$-seeds yielding potent seeds for propagation of Tau pathology in vivo, as demonstrated in this work, strongly fits with published genetic, pathological, clinical and biomarker data from patients. It presents as a compelling mechanism for $\mathrm{A} \beta$-induced Tau-pathology in $\mathrm{AD}$ and fits some peculiar findings in AD patients. Tau-pathology in EC and LC is present already early in life in most healthy people, but this early pathology does not spread beyond EC and LC and is not associated with clinical symptoms $[3,4,19,25,26,31$, $46,47,53,54]$. Hence, this Tau-pathology can be considered as rather "silent" or "non-aggressive" (yet a form of pre-existing pathological Tau). In $\mathrm{AD}$, pathological analysis, clinical analysis and biomarker data indicate that following accumulation of a high $\beta$-amyloid load in isocortical regions, Tau-pathology is propagated beyond EC/LC in a more aggressive way and with associated symptoms [3, 4, 19, 25, 26, 31, 46, 47, 53, 54]. Heterotypic seeding of Tau-pathology by $\mathrm{A} \beta$-seeds thereby provides a compelling molecular mechanism of $A \beta$-induced propagation of Taupathology in $\mathrm{AD}$, capable of resolving some peculiar findings in $\mathrm{AD}$ as further outlined.

Firstly, in $\mathrm{AD} A \beta$ and Tau-pathology progress in remarkably distinct spatio-temporal patterns according to Thal and
Braak staging $[3,54]$. Nevertheless, increasing $A \beta$ load in remote brain regions is generally accepted to drive propagation of Tau-pathology. Heterotypic induced Tau-aggregation by $\mathrm{A} \beta$-seeds is in line with this (1) spatial paradox, since this seeding process can occur along functional connections. Hence, $A \beta$-seeds, strongly accumulating in isocortical regions can via functional connections cross-seed Tau-pathology in EC to spread beyond EC. Prion-like seeding and propagation of Tau-aggregation has been demonstrated to occur between functionally connected brain regions $[1,6,16,17,22,28,39,44,51]$, reminiscent of the progression of Tau-pathology in $\mathrm{AD}[1,3,7,22,27,51]$. Interestingly, in vivo models of $A \beta$-induced Tau-pathology display features reminiscent of this prion-like seeding process $[2,13,40,50]$.

Secondly, it is intriguing that in $\mathrm{AD}$ brains, despite accumulation of high $\beta$-amyloid plaque load in isocortical brain regions, these regions remain (2) relatively spared from Tau-pathology in earlier stages of the disease [3, 54]. And instead, there is a (3) preferential propagation of Taupathology out of regions with pre-existing Tau-pathology, resulting in the very characteristic Braak staging. Intriguingly, we here demonstrate that $\mathrm{A} \beta$-seeded Tau-aggregation efficiently accelerates pre-existing Tau-pathology. This is also in line with findings in preclinical models, where no induction of Tau-aggregation is demonstrated in models expressing APP/PS1, or following injection of pre-aggregated $\mathrm{A} \beta$ without overexpression of Tau on the murine Tau background, in contrast to models overexpressing mutant Tau [49].

Thirdly, heterotypic seeding of Tau by $\mathrm{A} \beta$ could explain (4) conversion to more potent and aggressively propagating Tau-pathology beyond EC, following cross-seeding by A $\beta$-seeds. And finally, since (5) homotypic prion-like seeding occurs preferentially, initially $A \beta$ load will increase preferentially and exponentially in isocortical regions [54]. This exponentially increasing $A \beta$ load may thereby increase dramatically the probability of cross-seeding, inducing propagation of Tau-pathology, associated with the conversion to AD.

Taken together, heterotypic seeding of filamentous Tauaggregation by $\mathrm{A} \beta$ presents as a compelling mechanism for $A \beta$-induced Tau-pathology, capable of resolving some peculiar AD patient data. However, we do not exclude the potential involvement of other molecular and cellular processes in $\mathrm{A} \beta$-induced Tau-pathology, particularly neuronal signaling pathways and inflammation. The respective contribution of these mechanisms to $A \beta$-induced Tau-pathology in preclinical $\mathrm{AD}$ models and $\mathrm{AD}$ patients remains to be further analyzed.

In conclusion, $A \beta$-induced Tau-pathology is based on genetic data, biomarker data, clinical data and data from in vitro and in vivo models to be considered as a key 
a
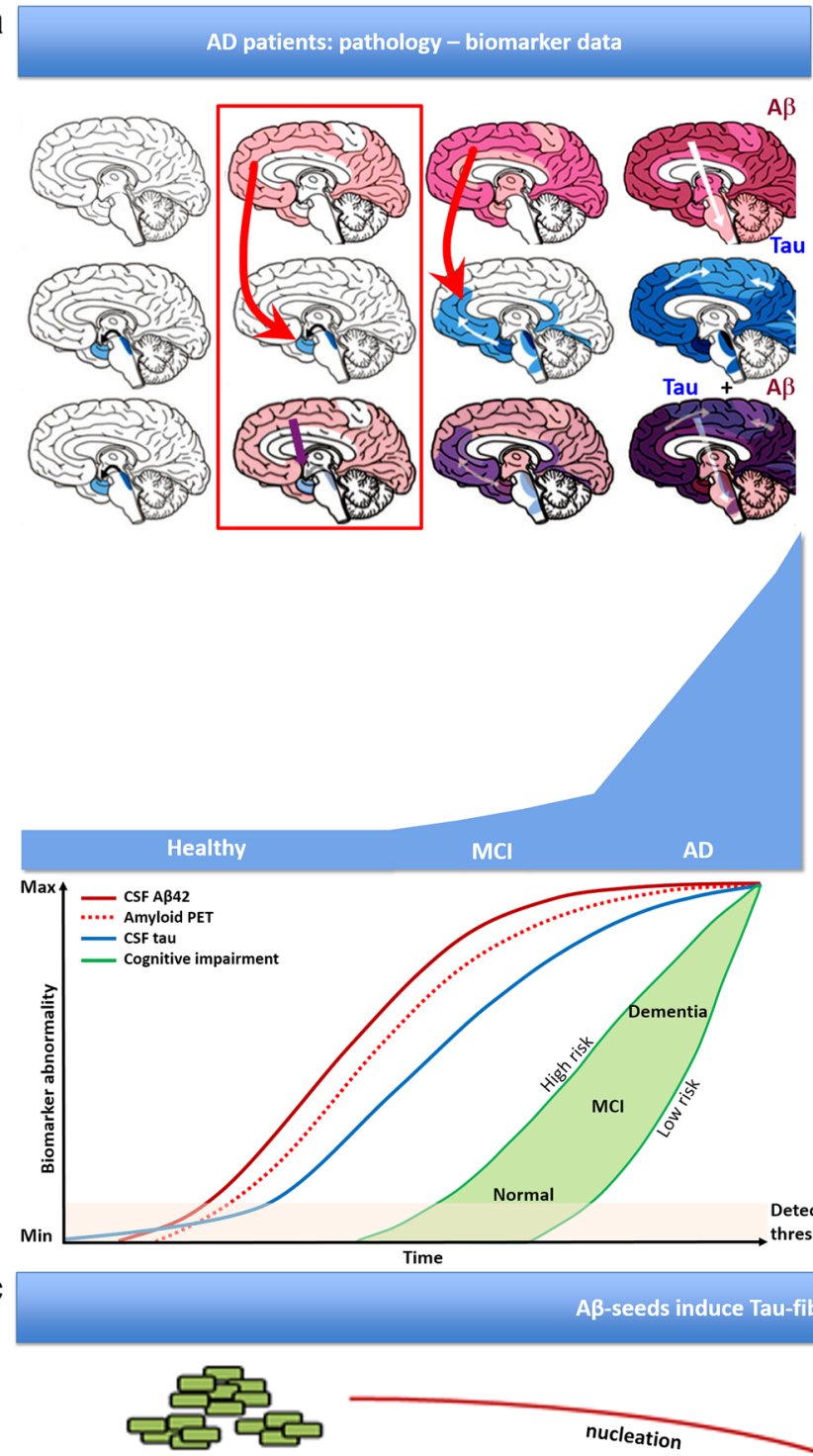

$A \beta$ aggregates

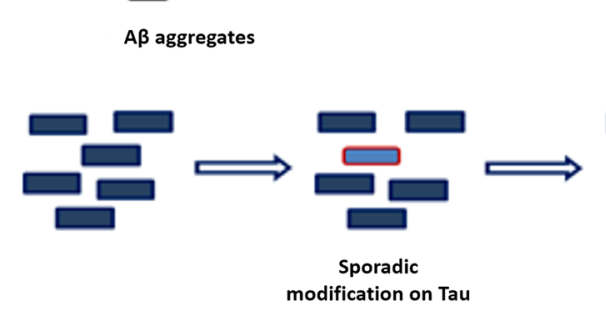

SLOW

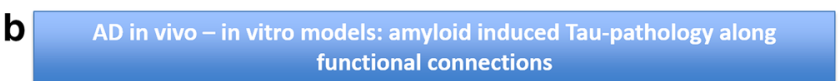

Injection of synthetic pre-aggregated $A \beta$ induced NFTs along functional

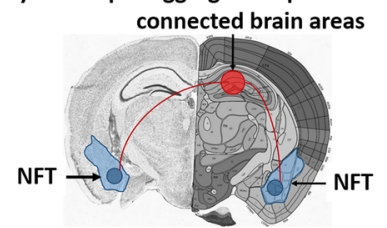

Gotz et al. 2001

Injection of mouse brain extracts containing pre-aggregated $A \beta$-induction of NFTs in functionally connected brain areas

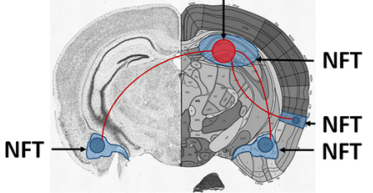

Bolmont et al. 2007

$A \beta$ induced Tau-pathology along functional connections

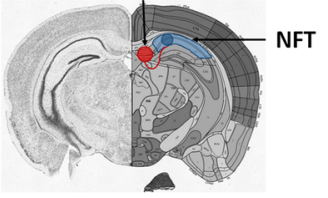

Stancu et al. 2014

Amyloid pathology drives Tau-pathology out of entorhinal cortex

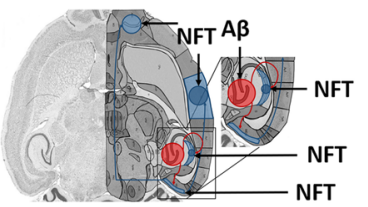

Pooler et al. 2015

3D neuronal culture - NFTs downstream of aggregated $A \beta$

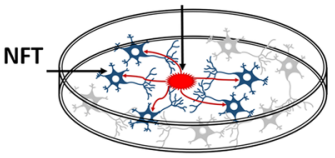

Choi et al. 2015 initiating mechanism in the pathogenesis of $\mathrm{AD}$, more particularly in the conversion from preclinical stages to MCI and $\mathrm{AD}[3,4,19,25,26,31,46,47,53,54]$. Unraveling the exact molecular mechanisms which can actually contribute to $A \beta$-induced Tau-pathology is required for design of novel therapeutic venues to halt or slow down conversion to AD. In this work we have proven the occurrence of heterotypic seeding of filamentous Tau-aggregation by $A \beta$ and further demonstrated the seeding and propagating potential of these newly identified species in vivo. We believe this 
4Fig. 9 Schematic summary of the work. a Clinical, genetic, pathological and biomarker data in patients support amyloid induced Tau-pathology and subsequent propagation beyond EC as a crucial process in the conversion from preclinical stages to AD. Schematic views are reproduced with minor modifications from publications of Jucker and Walker 2013 [27] and Jack et al. 2013 [25]. b. Data from different in vivo and in vitro models support $A \beta$ induced Taupathology to functionally connected brain regions following injections with (1) pre-aggregated synthetic $A \beta$ and (2) pre-aggregated $\mathrm{A} \beta$ from brain extracts from amyloid plaque displaying $\mathrm{AD}$ models and (3) AD patients, as well as in different preclinical AD models displaying $A \beta$ induced Tau-pathology. Schematic presentations of the results presented in previous papers are presented $[2,5,13,40$, $50]$. This process is reminiscent of the spatial dissociation of $A \beta$ and Tau-pathology in $\mathrm{AD}$, in which (pre-) aggregated $\mathrm{A} \beta$ aggravates Tau-pathology along functional connections [3, 54]. These findings are reminiscent of the prion-like propagation of Tau-pathology along functionally connected brain regions in Tau-models. A $\beta$-induced Taupathology could hence be driven by cross-seeding between $\mathrm{A} \beta$-seeds and Tau, similarly as proven for alpha-synuclein and Tau. $\mathbf{c}$ In this work, we demonstrate that pre-aggregated $\mathrm{A} \beta$ provides seeds for Taufibrillization by cross-seeding, providing potent seeds for inducing Tau-aggregation and propagation of Tau-pathology in Tau-transgenic mice in vivo. Without excluding involvement of other mechanisms, including inflammation and synaptic/neuronal processes, $A \beta$-induced Tau-aggregation by cross-seeding provides a compelling molecular model for $\mathrm{A} \beta$ induced Tau-pathology in preclinical models of $\mathrm{AD}$ and in $\mathrm{AD}$ patients, capable of resolving some particularities observed in AD patients-as outlined in "Discussion"

presents a very compelling mechanism for $A \beta$-induced Taupathology in preclinical models of $\mathrm{AD}$, and $\mathrm{AD}$ patients, which is in line with and capable of resolving some characteristic findings in $\mathrm{AD}$ patients.

Acknowledgments This work was supported by the Belgian Fonds National pour la Recherche Scientifique-Fonds de la Recherche Scientifique (FNRS-FRS; Qualified Researcher, Impulse Financing, Research Credits), by the Stellar project (Johnson and Johnson pharmaceutical companies), by Interuniversity Attraction Poles Programme-Belgian State-Belgian Science Policy, The Belgian Fonds de la Recherche Scientifique Médicale, by the Fondation Médicale Reine Elisabeth (F.M.R.E.), by the Institute for the Promotion of Innovation and by Science and Technology (IWT) in Flanders (IWTO\&O), Belgium.

\section{Compliance with ethical standards}

Conflict of interest The authors have no competing interest. A. Buist, K. Van Kolen, A. Verheyen and D. Moechars are employees of Janssen Pharmaceutical Companies of Johnson and Johnson.

Open Access This article is distributed under the terms of the Creative Commons Attribution 4.0 International License (http://creativecommons.org/licenses/by/4.0/), which permits unrestricted use, distribution, and reproduction in any medium, provided you give appropriate credit to the original author(s) and the source, provide a link to the Creative Commons license, and indicate if changes were made.

\section{References}

1. Ahmed Z, Cooper J, Murray TK, Garn K, McNaughton E, Clarke H, Parhizkar S, Ward MA, Cavallini A, Jackson S, Bose S, Clavaguera F, Tolnay M, Lavenir I, Goedert M, Hutton ML, O'Neill MJ (2014) A novel in vivo model of tau propagation with rapid and progressive neurofibrillary tangle pathology: the pattern of spread is determined by connectivity, not proximity. Acta Neuropathol 127:667-683

2. Bolmont T, Clavaguera F, Meyer-Luehmann M, Herzig MC, Radde R, Staufenbiel M, Lewis J, Hutton M, Tolnay M, Jucker M (2007) Induction of tau pathology by intracerebral infusion of amyloid-beta-containing brain extract and by amyloidbeta deposition in APP $\times$ Tau transgenic mice. Am J Pathol 171:2012-2020

3. Braak H, Braak E (1991) Neuropathological stageing of Alzheimer-related changes. Acta Neuropathol 82:239-259

4. Braak H, Del Tredici K (2011) The pathological process underlying Alzheimer's disease in individuals under thirty. Acta Neuropathol 121:171-181

5. Choi SH, Kim YH, Hebisch M, Sliwinski C, Lee S, D'Avanzo C, Chen J, Hooli B, Asselin C, Muffat J, Klee JB, Zhang C, Wainger BJ, Peitz M, Kovacs DM, Woolf CJ, Wagner SL, Tanzi RE, Kim DY (2014) A three-dimensional human neural cell culture model of Alzheimer's disease. Nature 515:274-278

6. Clavaguera F, Bolmont T, Crowther RA, Abramowski D, Frank S, Probst A, Fraser G, Stalder AK, Beibel M, Staufenbiel M, Jucker M, Goedert M, Tolnay M (2009) Transmission and spreading of tauopathy in transgenic mouse brain. Nat Cell Biol 11:909-913

7. de Calignon A, Polydoro M, Suarez-Calvet M, William C, Adamowicz DH, Kopeikina KJ, Pitstick R, Sahara N, Ashe KH, Carlson GA, Spires-Jones TL, Hyman BT (2012) Propagation of tau pathology in a model of early Alzheimer's disease. Neuron 73:685-697

8. Eisenberg D, Jucker M (2012) The amyloid state of proteins in human diseases. Cell 148:1188-1203

9. Fernandez MS (2014) Human IAPP amyloidogenic properties and pancreatic beta-cell death. Cell Calcium 56:416-427

10. Friedhoff P, Schneider A, Mandelkow EM, Mandelkow E (1998) Rapid assembly of Alzheimer-like paired helical filaments from microtubule-associated protein tau monitored by fluorescence in solution. Biochemistry 37:10223-10230

11. Giasson BI, Forman MS, Higuchi M, Golbe LI, Graves CL, Kotzbauer PT, Trojanowski JQ, Lee VM (2003) Initiation and synergistic fibrillization of tau and alpha-synuclein. Science 300:636-640

12. Goedert M (2015) NEURODEGENERATION. Alzheimer's and Parkinson's diseases: the prion concept in relation to assembled Abeta, tau, and alpha-synuclein. Science 349:1255555

13. Gotz J, Chen F, van DJ, Nitsch RM (2001) Formation of neurofibrillary tangles in P3011 tau transgenic mice induced by Abeta 42 fibrils. Science 293:1491-1495

14. Guerrero-Muñoz MJ, Castillo-Carranza DL, Krishnamurthy $S$, Paulucci-Holthauzen AA, Sengupta U, Lasagna-Reeves CA, Ahmad Y, Jackson GR, Kayed R (2014) Amyloid- $\beta$ oligomers as a template for secondary amyloidosis in Alzheimer's disease. Neurobiol Dis 71:14-23

15. Guo JL, Covell DJ, Daniels JP, Iba M, Stieber A, Zhang B, Riddle DM, Kwong LK, Xu Y, Trojanowski JQ, Lee VM (2013) Distinct alpha-synuclein strains differentially promote tau inclusions in neurons. Cell 154:103-117 
16. Guo JL, Lee VM (2011) Seeding of normal Tau by pathological Tau conformers drives pathogenesis of Alzheimer-like tangles. J Biol Chem 286:15317-15331

17. Guo JL, Lee VM (2014) Cell-to-cell transmission of pathogenic proteins in neurodegenerative diseases. Nat Med 20:130-138

18. Guo JP, Arai T, Miklossy J, McGeer PL (2006) Abeta and tau form soluble complexes that may promote self aggregation of both into the insoluble forms observed in Alzheimer's disease. Proc Natl Acad Sci USA 103:1953-1958

19. Hardy J, Selkoe DJ (2002) The amyloid hypothesis of Alzheimer's disease: progress and problems on the road to therapeutics. Science 297:353-356

20. Heraud C, Goufak D, Ando K, Leroy K, Suain V, Yilmaz Z, De Decker R, Authelet M, Laporte V, Octave JN, Brion JP (2014) Increased misfolding and truncation of tau in APP/PS1/tau transgenic mice compared to mutant tau mice. Neurobiol Dis 62:100-112

21. Hurtado DE, Molina-Porcel L, Iba M, Aboagye AK, Paul SM, Trojanowski JQ, Lee VM (2010) A $\{$ beta $\}$ accelerates the spatiotemporal progression of tau pathology and augments tau amyloidosis in an Alzheimer mouse model. Am J Pathol 177:1977-1988

22. Iba M, Guo JL, McBride JD, Zhang B, Trojanowski JQ, Lee VM (2013) Synthetic tau fibrils mediate transmission of neurofibrillary tangles in a transgenic mouse model of Alzheimer's-like tauopathy. J Neurosci Off J Soc Neurosci 33:1024-1037

23. Ittner LM, Gotz J (2011) Amyloid-beta and tau-a toxic pas de deux in Alzheimer's disease. Nat Rev Neurosci 12:65-72

24. Ittner LM, Ke YD, Delerue F, Bi M, Gladbach A, van Eersel J, Wolfing H, Chieng BC, Christie MJ, Napier IA, Eckert A, Staufenbiel M, Hardeman E, Gotz J (2010) Dendritic function of tau mediates amyloid-beta toxicity in Alzheimer's disease mouse models. Cell 142:387-397

25. Jack CR Jr, Knopman DS, Jagust WJ, Petersen RC, Weiner MW, Aisen PS, Shaw LM, Vemuri P, Wiste HJ, Weigand SD, Lesnick TG, Pankratz VS, Donohue MC, Trojanowski JQ (2013) Tracking pathophysiological processes in Alzheimer's disease: an updated hypothetical model of dynamic biomarkers. Lancet Neurol 12:207-216

26. Jack CR Jr, Knopman DS, Jagust WJ, Shaw LM, Aisen PS, Weiner MW, Petersen RC, Trojanowski JQ (2010) Hypothetical model of dynamic biomarkers of the Alzheimer's pathological cascade. Lancet Neurol 9:119-128

27. Jucker M, Walker LC (2013) Self-propagation of pathogenic protein aggregates in neurodegenerative diseases. Nature 501:45-51

28. Kfoury N, Holmes BB, Jiang H, Holtzman DM, Diamond MI (2012) Trans-cellular propagation of Tau aggregation by fibrillar species. J Biol Chem 287:19440-19451

29. Lewis J, Dickson DW, Lin WL, Chisholm L, Corral A, Jones G, Yen SH, Sahara N, Skipper L, Yager D, Eckman C, Hardy J, Hutton M, McGowan E (2001) Enhanced neurofibrillary degeneration in transgenic mice expressing mutant tau and APP. Science 293:1487-1491

30. Luk KC, Kehm V, Carroll J, Zhang B, O'Brien P, Trojanowski JQ, Lee VM (2012) Pathological alpha-synuclein transmission initiates Parkinson-like neurodegeneration in nontransgenic mice. Science 338:949-953

31. Mann DM, Hardy J (2013) Amyloid or tau: the chicken or the egg? Acta Neuropathol 126:609-613

32. Mares RE, Melendez-Lopez SG, Ramos MA (2011) Acid-denatured Green Fluorescent Protein (GFP) as model substrate to study the chaperone activity of protein disulfide isomerase. Int $\mathrm{J}$ Mol Sci 12:4625-4636

33. Meyer-Luehmann M, Coomaraswamy J, Bolmont T, Kaeser S, Schaefer C, Kilger E, Neuenschwander A, Abramowski D, Frey P, Jaton AL, Vigouret JM, Paganetti P, Walsh DM, Mathews PM,
Ghiso J, Staufenbiel M, Walker LC, Jucker M (2006) Exogenous induction of cerebral beta-amyloidogenesis is governed by agent and host. Science 313:1781-1784

34. Miller Y, Ma B, Nussinov R (2011) Synergistic interactions between repeats in Tau protein and $\mathrm{A} \beta$ amyloids may be responsible for accelerated aggregation via polymorphic states. Biochemistry 50:5172-5181

35. Morales R, Moreno-Gonzalez I, Soto C (2013) Cross-seeding of misfolded proteins: implications for etiology and pathogenesis of protein misfolding diseases. PLoS Pathog 9:e1003537

36. Oddo S, Billings L, Kesslak JP, Cribbs DH, LaFerla FM (2004) Abeta immunotherapy leads to clearance of early, but not late, hyperphosphorylated tau aggregates via the proteasome. Neuron 43:321-332

37. Paxinos G, Franklin K (2001) The mouse brain in stereotaxic coordinates. Academic, San Diego

38. Peelaerts W, Bousset L, Van der Perren A, Moskalyuk A, Pulizzi R, Giugliano M, Van den Haute C, Melki R, Baekelandt V (2015) Alpha-synuclein strains cause distinct synucleinopathies after local and systemic administration. Nature 522:340-344

39. Peeraer E, Bottelbergs A, Van Kolen K, Stancu I-C, Vasconcelos B, Mahieu M, Duytschaever H, Ver Donck L, Torremans A, Sluydts E, Van Acker N, Kemp JA, Mercken M, Brunden KR, Trojanowski JQ, Dewachter I, Lee VMY, Moechars D (2015) Intracerebral injection of preformed synthetic tau fibrils initiates widespread tauopathy and neuronal loss in the brains of tau transgenic mice. Neurobiol Dis 73:83-95

40. Pooler AM, Polydoro M, Maury EA, Nicholls SB, Reddy SM, Wegmann S, William C, Saqran L, Cagsal-Getkin O, Pitstick R, Beier DR, Carlson GA, Spires-Jones TL, Hyman BT (2015) Amyloid accelerates tau propagation and toxicity in a model of early Alzheimer's disease. Acta Neuropathol Commun 3:14

41. Postina R, Schroeder A, Dewachter I, Bohl J, Schmitt U, Kojro E, Prinzen C, Endres K, Hiemke C, Blessing M, Flamez P, Dequenne A, Godaux E, van Leuven F, Fahrenholz F (2004) A disintegrin-metalloproteinase prevents amyloid plaque formation and hippocampal defects in an Alzheimer disease mouse model. J Clin Investig 113:1456-1464

42. Rhoades E, Agarwal J, Gafni A (2000) Aggregation of an amyloidogenic fragment of human islet amyloid polypeptide. Biochim Biophys Acta 1476:230-238

43. Rhoades E, Gafni A (2003) Micelle formation by a fragment of human islet amyloid polypeptide. Biophys J 84:3480-3487

44. Sanders DW, Kaufman SK, DeVos SL, Sharma AM, Mirbaha H, Li A, Barker SJ, Foley AC, Thorpe JR, Serpell LC, Miller TM, Grinberg LT, Seeley WW, Diamond MI (2014) Distinct tau prion strains propagate in cells and mice and define different tauopathies. Neuron 82:1271-1288

45. Saul A, Sprenger F, Bayer TA, Wirths O (2013) Accelerated tau pathology with synaptic and neuronal loss in a novel triple transgenic mouse model of Alzheimer's disease. Neurobiol Aging 34:2564-2573

46. Selkoe DJ (2011) Alzheimer's disease. Cold Spring Harb Perspect Biol 3:a004457

47. Serrano-Pozo A, Frosch MP, Masliah E, Hyman BT (2011) Neuropathological alterations in Alzheimer disease. Cold Spring Harb Perspect Med 1:a006189

48. Spires-Jones TL, Hyman BT (2014) The intersection of amyloid beta and tau at synapses in Alzheimer's disease. Neuron 82:756-771

49. Stancu I-C, Vasconcelos B, Terwel D, Dewachter I (2014) Models of $\beta$-amyloid induced Tau-pathology: the long and "folded" road to understand the mechanism. Mol Neurodegener 9:51

50. Stancu IC, Ris L, Vasconcelos B, Marinangeli C, Goeminne L, Laporte V, Haylani LE, Couturier J, Schakman O, Gailly P, Pierrot N, Kienlen-Campard P, Octave JN, Dewachter I (2014) 
Tauopathy contributes to synaptic and cognitive deficits in a murine model for Alzheimer's disease. FASEB J Off Publ Fed Am Soc Exp Biol 28:2620-2631

51. Stancu IC, Vasconcelos B, Ris L, Wang P, Villers A, Peeraer E, Buist A, Terwel D, Baatsen P, Oyelami T, Pierrot N, Casteels C, Bormans G, Kienlen-Campard P, Octave JN, Moechars D, Dewachter I (2015) Templated misfolding of Tau by prion-like seeding along neuronal connections impairs neuronal network function and associated behavioral outcomes in Tau transgenic mice. Acta Neuropathol 129:875-894

52. Stine WB Jr, Dahlgren KN, Krafft GA, LaDu MJ (2003) In vitro characterization of conditions for amyloid-beta peptide oligomerization and fibrillogenesis. J Biol Chem 278:11612-11622

53. Tanzi RE (2012) The genetics of Alzheimer disease. Cold Spring Harb Perspect Med 2:a006296

54. Thal DR, Rub U, Orantes M, Braak H (2002) Phases of A betadeposition in the human brain and its relevance for the development of AD. Neurology 58:1791-1800
55. von Bergen $\mathrm{M}$, Friedhoff $\mathrm{P}$, Biernat J, Heberle J, Mandelkow EM, Mandelkow E (2000) Assembly of tau protein into Alzheimer paired helical filaments depends on a local sequence motif ((306)VQIVYK(311)) forming beta structure. Proc Natl Acad Sci USA 97:5129-5134

56. Walker LC, Jucker M (2015) Neurodegenerative diseases: expanding the prion concept. Annu Rev Neurosci 38:87-103

57. Waxman EA, Giasson BI (2011) Induction of intracellular tau aggregation is promoted by alpha-synuclein seeds and provides novel insights into the hyperphosphorylation of tau. J Neurosci Off J Soc Neurosci 31:7604-7618

58. Yoshiyama Y, Higuchi M, Zhang B, Huang SM, Iwata N, Saido TC, Maeda J, Suhara T, Trojanowski JQ, Lee VM (2007) Synapse loss and microglial activation precede tangles in a P301S tauopathy mouse model. Neuron 53:337-351 